\title{
Ninhydrin Revisited: Quantitative Chirality Recognition of Amines and Amino Alcohols Based on Nondestructive Dynamic Covalent Chemistry
}

\author{
Samantha L. Pilicer and Christian Wolf* \\ Department of Chemistry, Georgetown University, Washington, DC 20057, USA.
}

\section{$\underline{\text { Tables of Contents }}$}

1. General information

2. Chiroptical sensing of amines

3. Chiroptical sensing of amino alcohols

4. Chiroptical sensing of diamines

5. Optimization and mechanistic studies

5.1 NMR analysis

5.2 UV analysis

5.3 CD analysis confirming stoichiometric binding

5.4 Reaction time and product stability

5.5 Sensing solvent selection

5.6 Anisotropy g-factor and molar ellipticity spectra

5.7 Dynamic covalent chemistry and equilibration study

6. Quantitative ee determination

7. References
S2

S3

S7

S13

S15

S15

S22

S25

S26

S30

S30

S37

S39

S40 


\section{General information}

All reagents and solvents were commercially available and used without purification. Ninhydrin hydrate, A, was obtained from Alfa Aesar, and all chiral analytes were purchased in their enantiopure forms from Sigma Aldrich or Combi-Blocks and used as is. The sensing reactions were carried out using anhydrous solvents. NMR spectra were obtained at $400 \mathrm{MHz}\left({ }^{1} \mathrm{H}-\mathrm{NMR}\right)$ and $100 \mathrm{MHz}\left({ }^{13} \mathrm{C}-\mathrm{NMR}\right)$ using $\mathrm{DMSO}-\mathrm{d}_{6}$ as solvent.

All CD experiments were conducted using acetonitrile as bulk solvent at $1.1 \mathrm{mM}$ analyte concentration unless indicated otherwise. The general scope of the ninhydrin chiroptical sensing was evaluated using stoichiometric amounts of chemically diverse primary amines and amino alcohols. All CD spectra were collected with a standard sensitivity of $100 \mathrm{mdeg}$, a data pitch of $1.0 \mathrm{~nm}$, a band width of $1.0 \mathrm{~nm}$, a scanning speed of $500 \mathrm{~nm} \mathrm{~min}$ mand $^{-1}$ a response of $1 \mathrm{~s}$ using a quartz cuvette (10 $\mathrm{mm}$ path length). Scans were corrected using a binomial smoothing function (15x). The chiral substrates surveyed do not produce a CD profile above $250 \mathrm{~nm}$ at the experimental conditions in the absence of the ninhydrin sensor. UV/Vis spectroscopy was conducted using a dual-beam Cary 50 spectrophotometer in bulk acetonitrile solvent with a $4800 \mathrm{~nm} / \mathrm{min}$ scan rate, $1.00 \mathrm{~nm}$ data interval, and averaged scan time of 0.0125 seconds. 


\section{Chiroptical sensing of amines}

A solution of ninhydrin hydrate using anhydrous DMSO was prepared and aliquots were distributed into reaction vials $(1.00 \mathrm{~mL}, 5.00 \mathrm{mM})$. Concentrated solutions of the chiral analytes dissolved in DMSO were prepared in parallel and stoichiometric equivalents $(0.005 \mathrm{mmol})$ were added to the vials. After 20 minutes, CD measurements were taken by diluting the $5.00 \mathrm{mM}$ solutions with ACN as indicated below. The final concentrations of the samples subjected to CD analysis are reported below.<smiles>CCC(N)c1ccccc1</smiles><smiles>CC(N)c1cccnc1</smiles><smiles>CC(N)c1cccc2ccccc12</smiles><smiles>CC(N)c1ccc2ccccc2c1</smiles><smiles>CC(C)C(C)N</smiles><smiles>CC(N)C(C)(C)C</smiles><smiles>CC(N)C1CCCCC1</smiles><smiles>CC1CCCN1</smiles>

Figure S1. Structures of amine analytes tested.

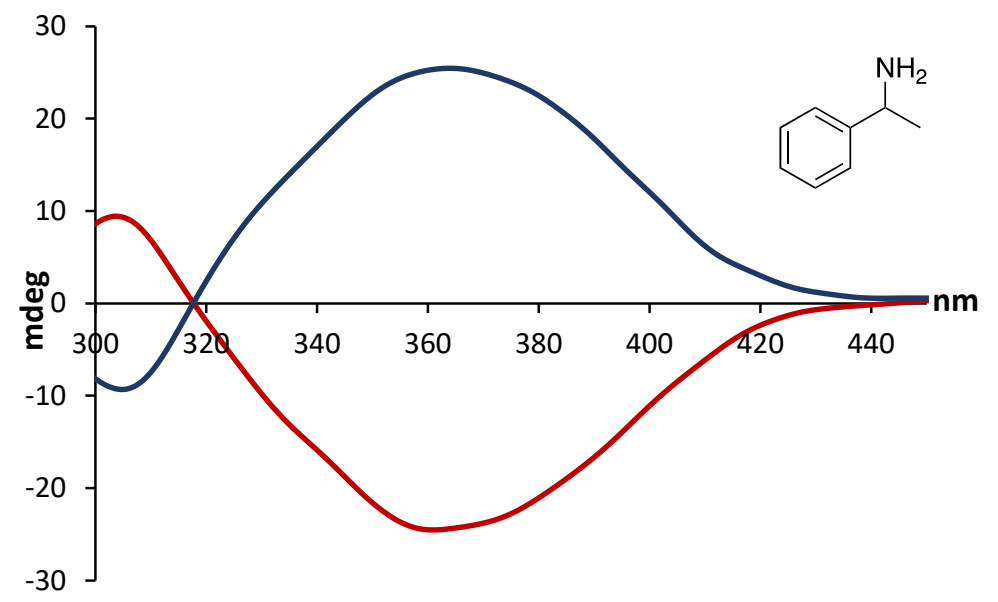

Figure S2. Sensing of with $(R)$-1-phenylethan-1-amine (red) and $(S)$-1-phenylethan-1-amine (blue) with $\mathrm{A}$ at $1.1 \mathrm{mM}$. 


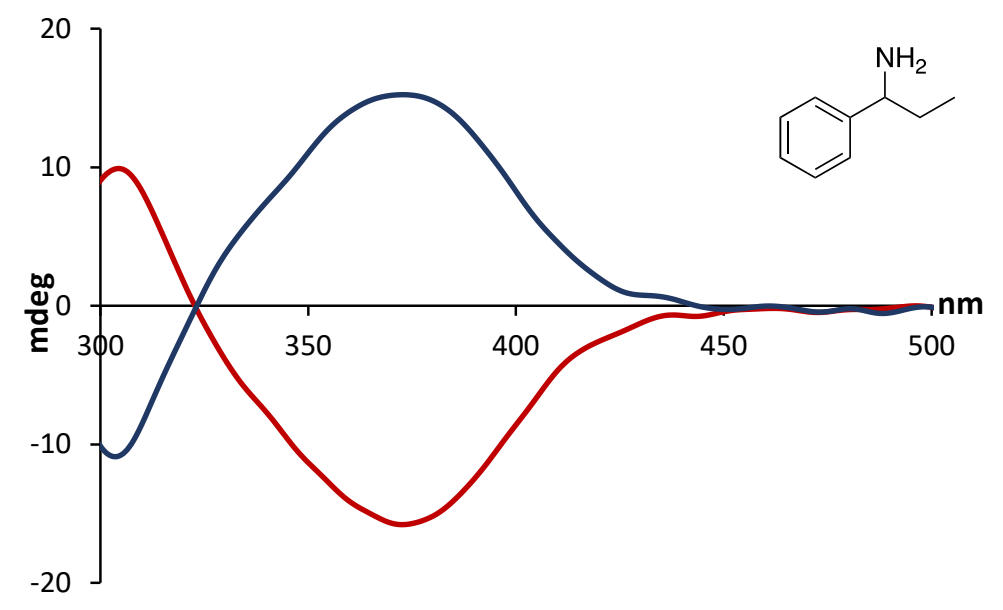

Figure S3. Sensing of with $(R)$-1-phenylpropan-1-amine (red) and (S)-1-phenylpropan-1-amine (blue) with $\mathrm{A}$ at $1.1 \mathrm{mM}$.

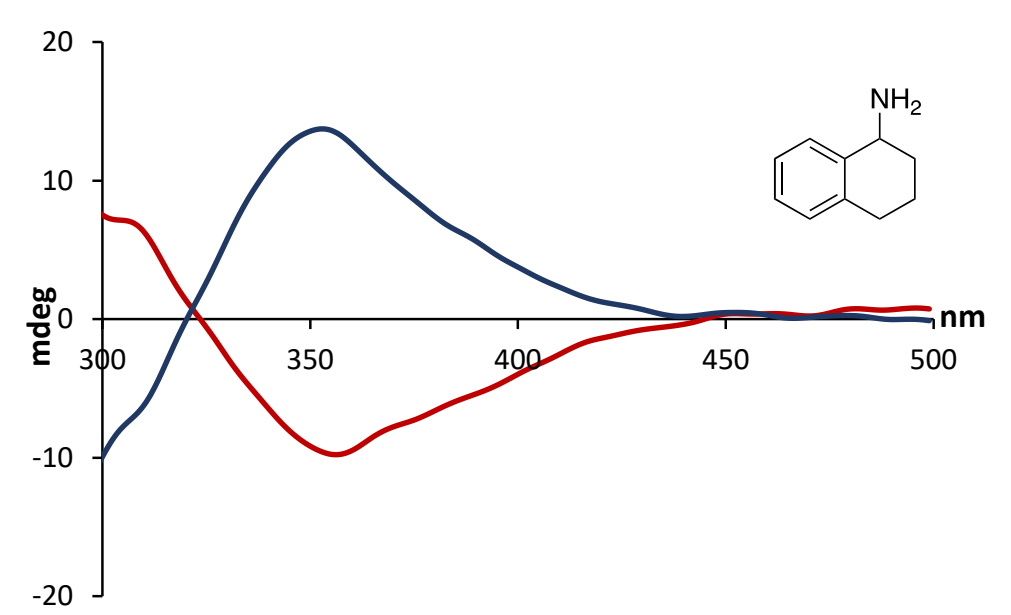

Figure S4. Sensing of $(R)-1,2,3,4$-tetrahydronaphthalen-1-amine (red) and (S)-1,2,3,4tetrahydronaphthalen-1-amine (blue) with $\mathbf{A}$ at $1.1 \mathrm{mM}$.

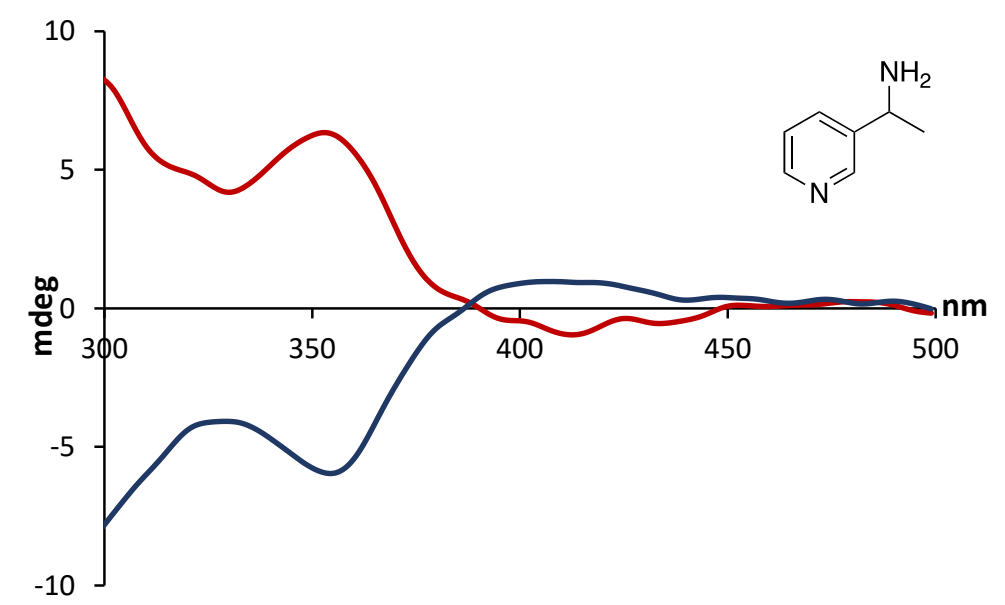

Figure S5. Sensing of $(R)$-1-(pyridin-3-yl)ethan-1-amine (red) and (S)-1-(pyridin-3-yl)ethan-1amine (blue) with $\mathbf{A}$ at $1.23 \mathrm{mM}$. 


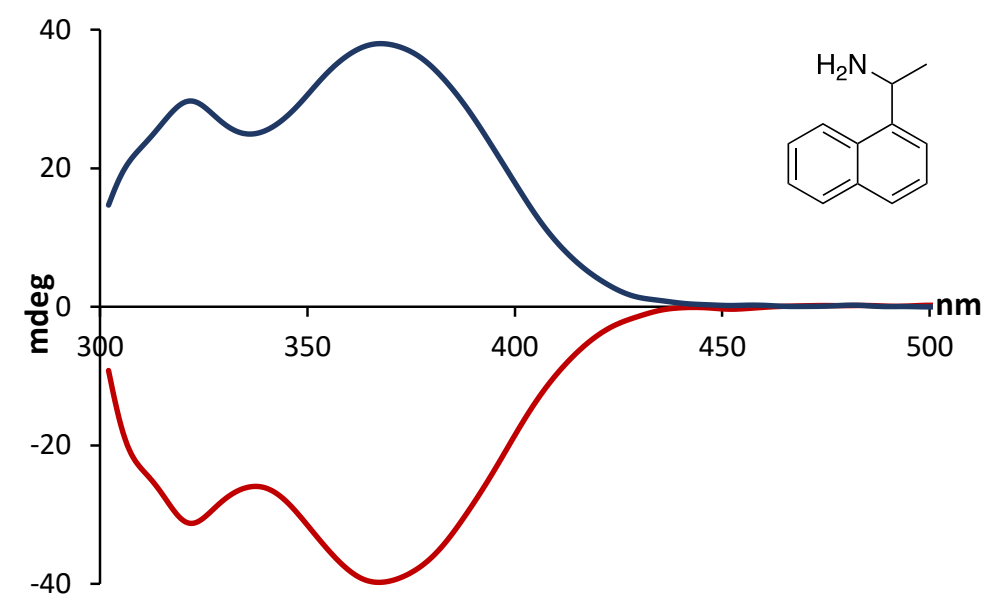

Figure S6. Sensing of $(R)$-1-(naphthalen-1-yl)ethan-1-amine (red) and (S)- 1-(naphthalen-1yl)ethan-1-amine (blue) with $A$ at $1.1 \mathrm{mM}$.

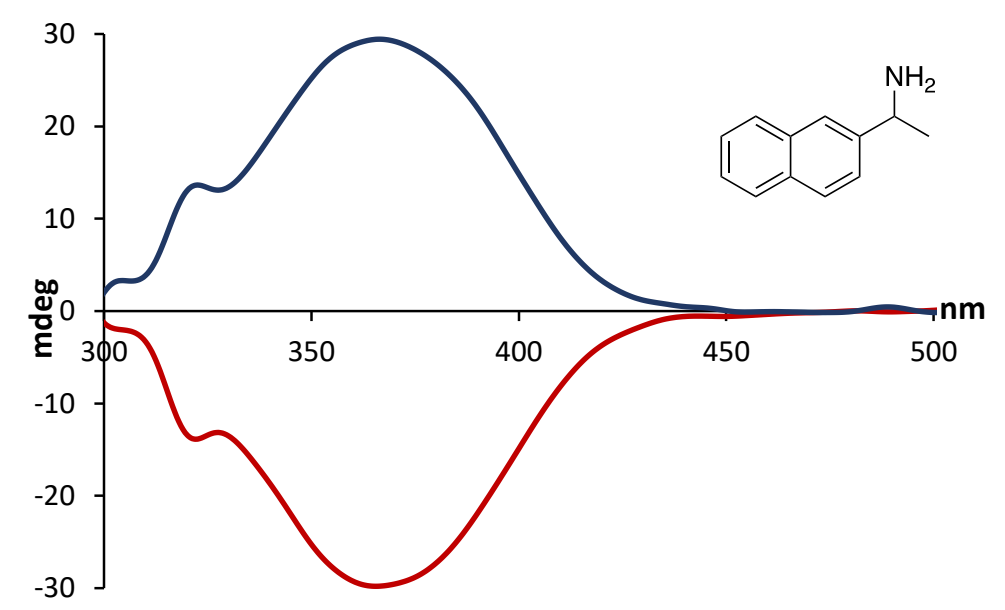

Figure S7. Sensing of (R)-1-(naphthalen-2-yl)ethan-1-amine (red) and (S)- 1-(naphthalen-2$\mathrm{yl}$ )ethan-1-amine (blue) with $\mathbf{A}$ at $1.1 \mathrm{mM}$.

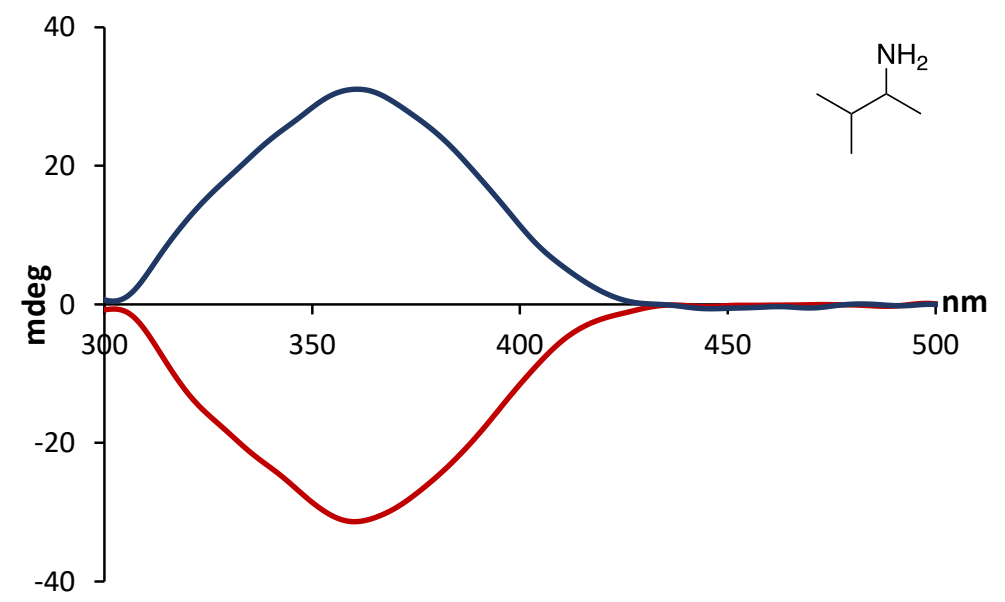

Figure S8. Sensing of (R)-3-methyl-butan-2-amine (red) and (S)-3-methyl-butan-2-amine (blue) with $A$ at $1.1 \mathrm{mM}$. 


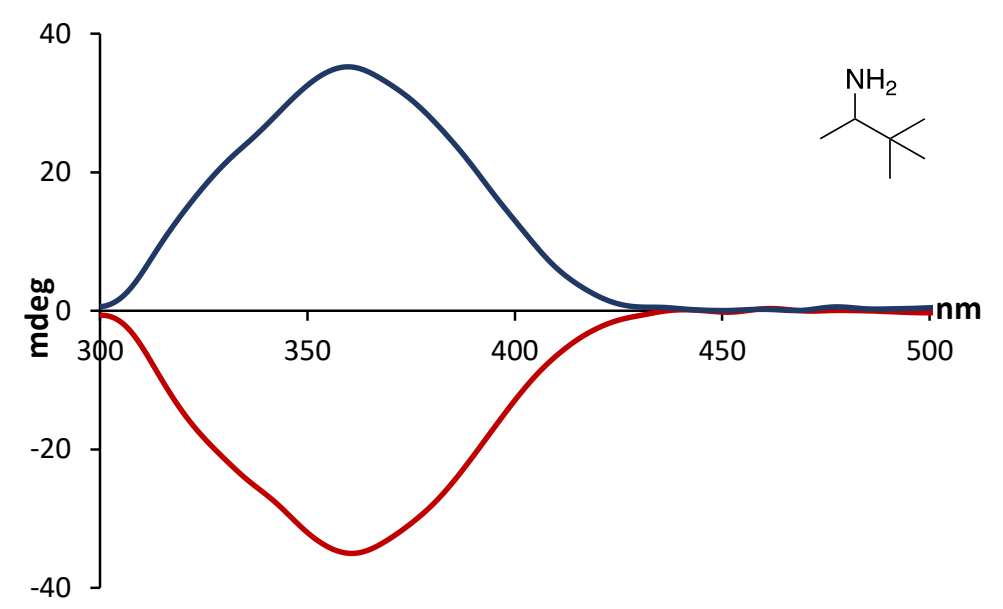

Figure S9. Sensing of $(R)$-3,3-dimethylbutan-2-amine (red) and (S)-3,3-dimethylbutan-2-amine (blue) with $\mathbf{A}$ at $1.1 \mathrm{mM}$.

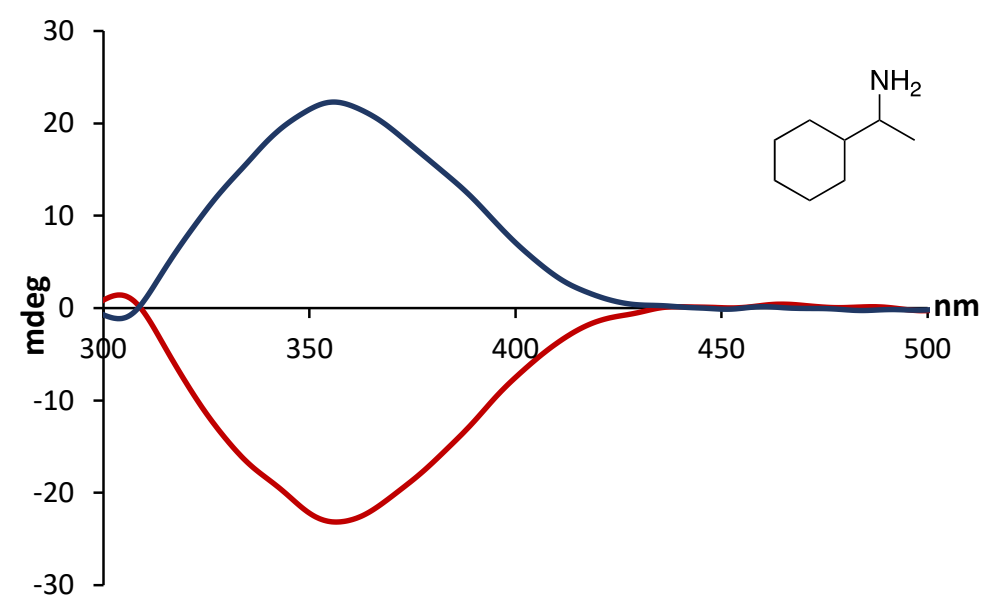

Figure S10. Sensing of (R)-1-cyclohexylethan-1-amine (red) and (S)-1- cyclohexylethan-1-amine (blue) with $\mathbf{A}$ at $1.1 \mathrm{mM}$.

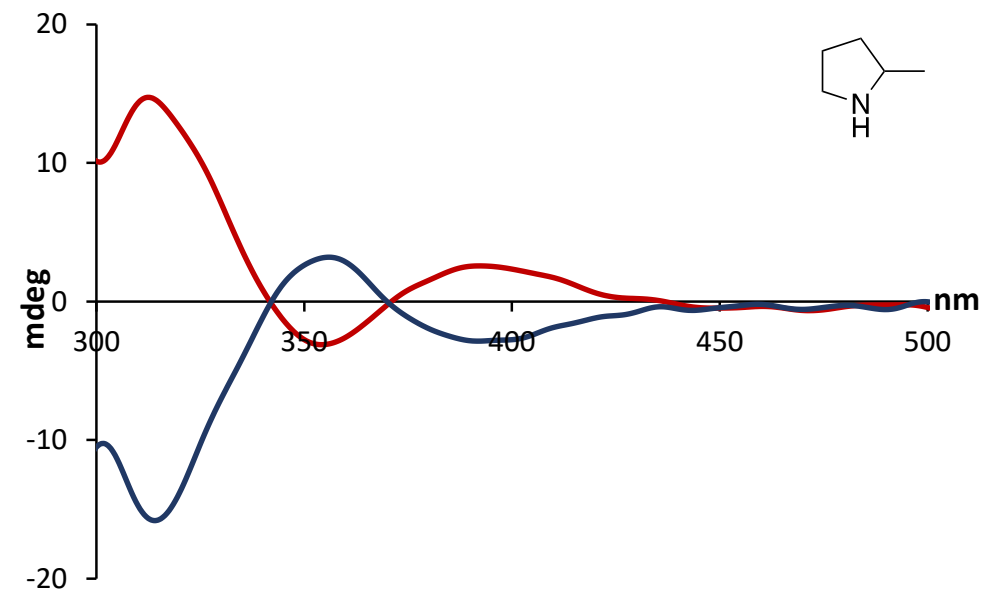

Figure S11. Sensing of (R)-2-methylpyrollidine (red) and (S)-2-methylpyrollidine (blue) with A at $1.1 \mathrm{mM}$. 


\section{Chiroptical sensing of amino alcohols}

A solution of ninhydrin hydrate using anhydrous DMSO was prepared and aliquots were distributed into reaction vials $(1.00 \mathrm{~mL}, 5.00 \mathrm{mM})$. Concentrated solutions of the chiral analytes dissolved in DMSO were prepared in parallel and stoichiometric equivalents $(0.005 \mathrm{mmol})$ were added to the vials. After 20 minutes, CD measurements were taken by diluting the $5.00 \mathrm{mM}$ solutions with ACN. The final concentrations of the samples subjected to CD analysis are reported below.<smiles>C[C@H](N)[C@H](O)c1ccccc1</smiles><smiles>NC(c1ccccc1)c1ccccc1</smiles><smiles>N[C@@H](c1ccccc1)[C@H](O)c1ccccc1</smiles><smiles>OC(CNCc1ccccc1)c1ccccc1</smiles><smiles>N[C@@H]1c2ccccc2CC1O</smiles><smiles>NC1c2ccccc2CC1O</smiles><smiles>NC1CCCC[C@H]1O</smiles><smiles>NC1CCCCC1O</smiles><smiles>C[C](N)CO</smiles><smiles>NCC(O)CO</smiles><smiles>CSCCC(N)CO</smiles><smiles>OCC1CCCN1</smiles>

Figure S12. Structures of amino alcohol analytes tested.

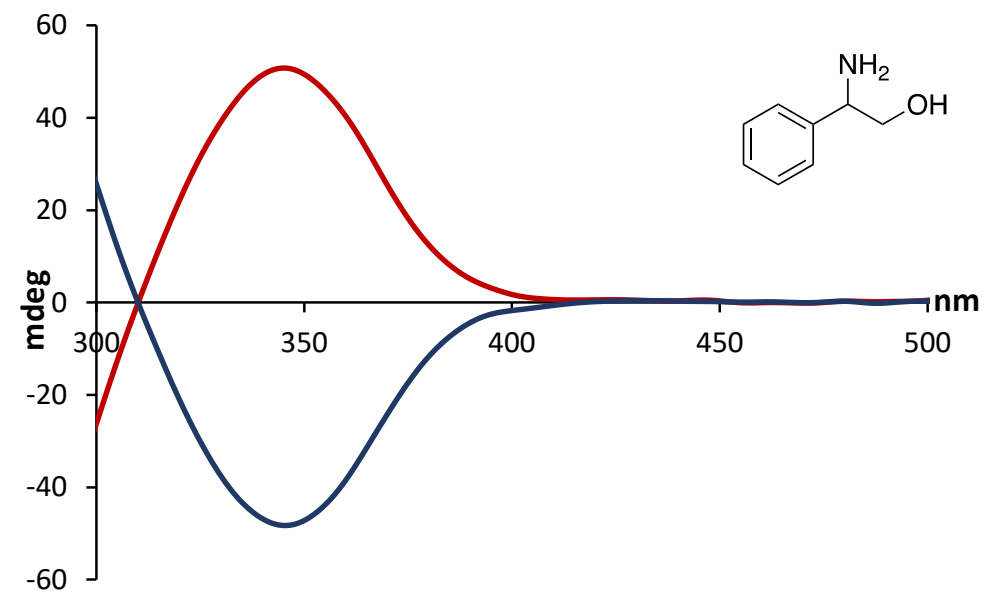

Figure S13. Sensing of $(R)$-2-amino-2-phenylethan-1-ol (red) and (S)-2-amino-2-phenylethan-1ol (blue) with $A$ at $1.1 \mathrm{mM}$. 


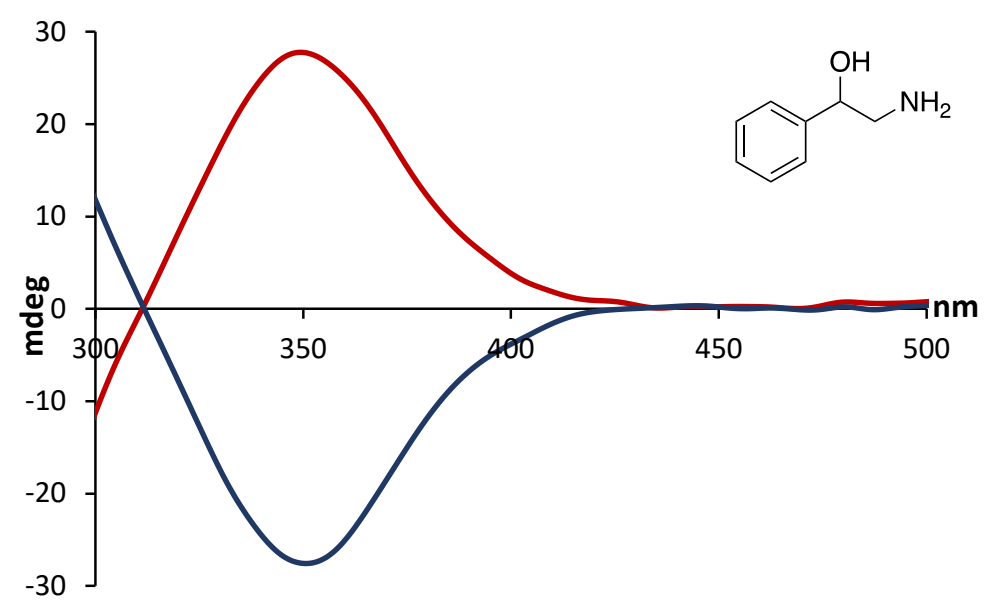

Figure S14. Sensing of $(R)$-2-amino-1-phenylethan-1-ol (red) and (S)-2-amino-1-phenylethan-1ol (blue) with $\mathrm{A}$ at $1.1 \mathrm{mM}$.

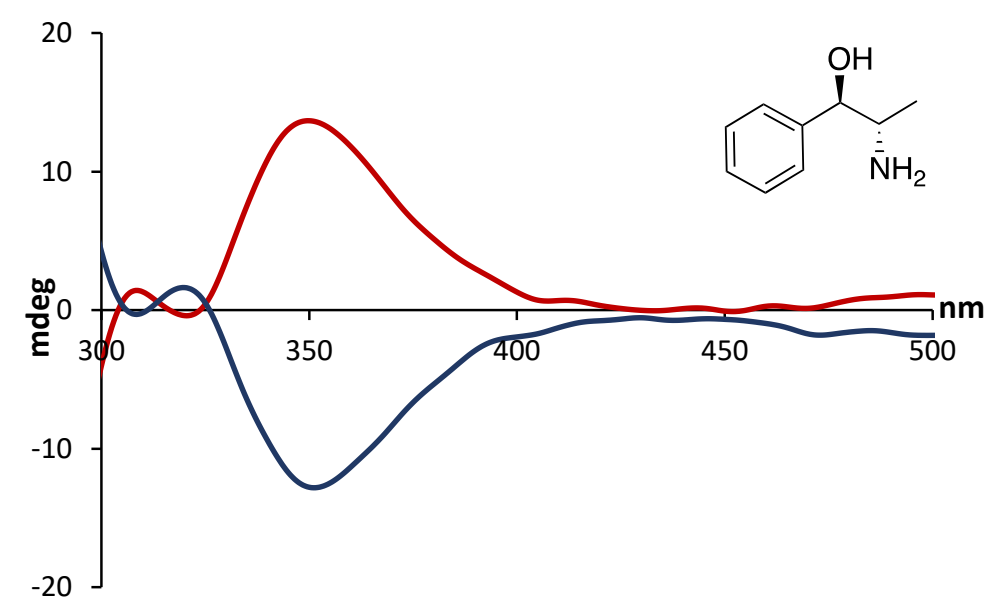

Figure S15. Sensing of (1R,2S)-2-amino-1-phenylpropan-1-ol (red) and (1S,2R)-2-amino-1phenylpropan-1-ol (blue) with $\mathbf{A}$ at $1.1 \mathrm{mM}$ (one enantiomeric structure shown).

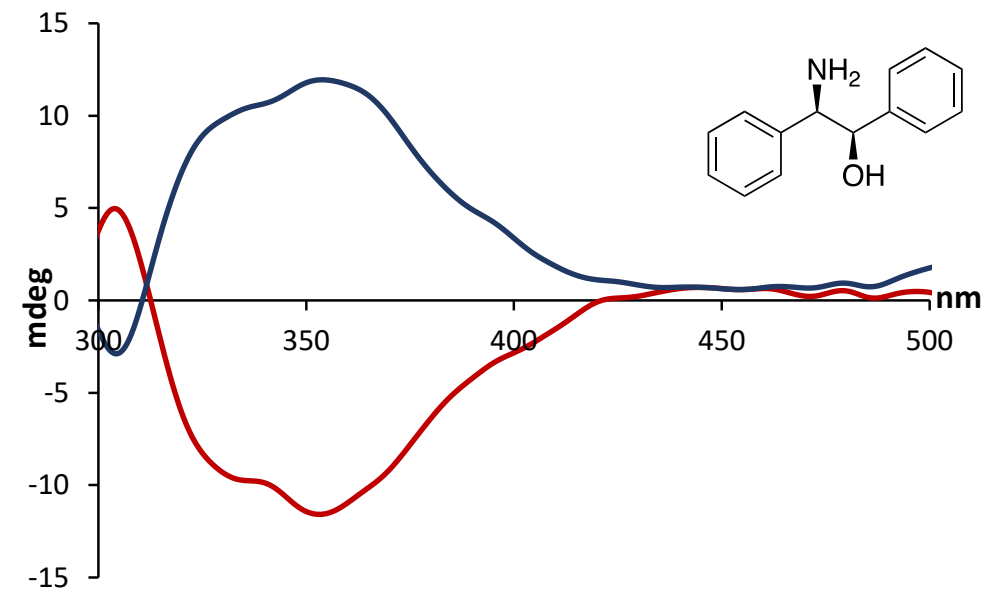

Figure S16. Sensing of (1R,2R)-2-amino-1,2-diphenylethan-1-ol (red) and (1S,2S)-2-amino-1,2diphenylethan-1-ol (blue) with $\mathbf{A}$ at $1.1 \mathrm{mM}$ (one enantiomeric structure shown). 


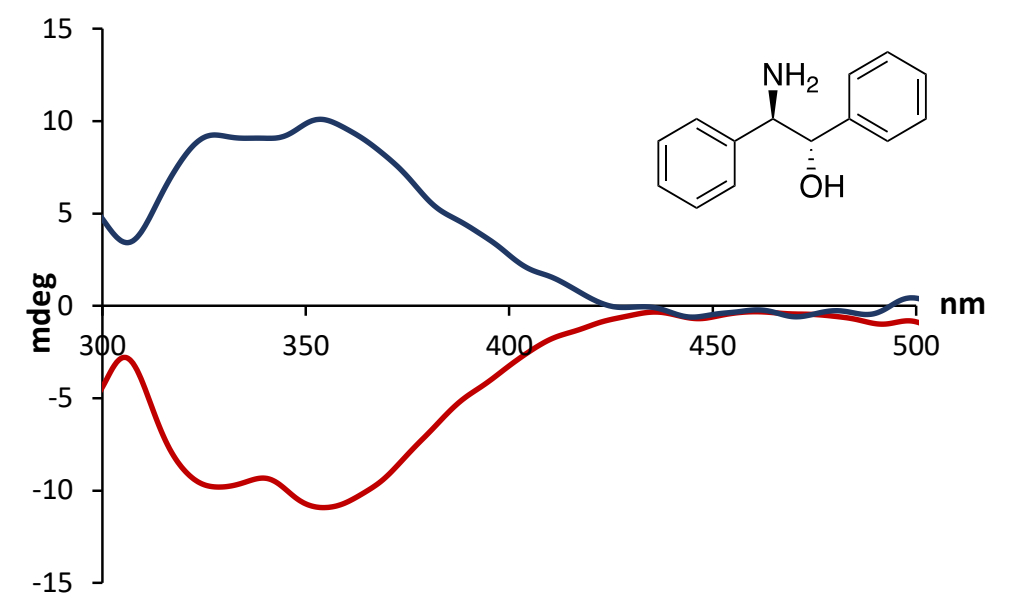

Figure S17. Sensing of (1R,2S)-2-amino-1,2-diphenylethan-1-ol (red) and (1S,2R)-2-amino-1,2diphenylethan-1-ol (blue) with $\mathbf{A}$ at $1.1 \mathrm{mM}$ (one enantiomeric structure shown).

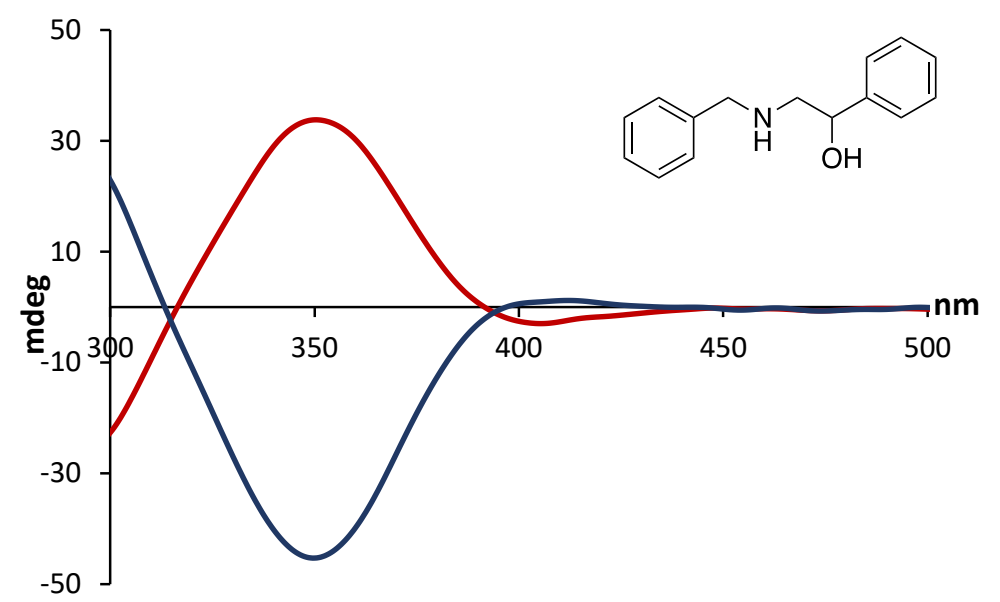

Figure S18. Sensing of (R)-2-(benzylamino)-1-phenylethan-1-ol (red) and (S)-2-(benzylamino)-1phenylethan-1-ol (blue) with $A$ at $0.55 \mathrm{mM}$.

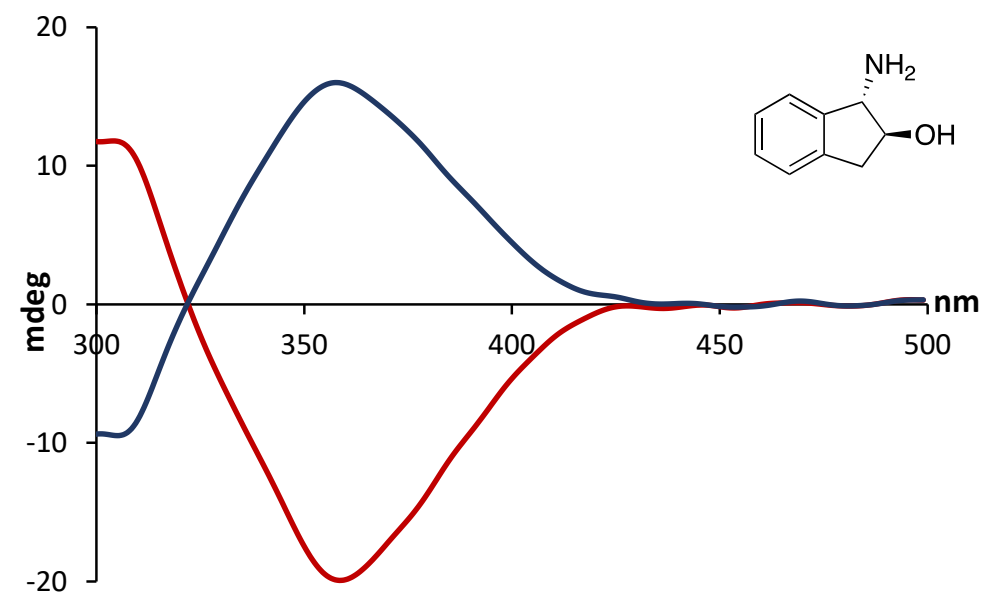

Figure S19. Sensing of $(1 R, 2 R)$-1-amino-2,3-dihydro- $1 H$-inden-2-ol (red) and (1S,2S)-1-amino2,3-dihydro- $1 H$-inden-2-ol (blue) with $\mathbf{A}$ at $0.55 \mathrm{mM}$ (one enantiomeric structure shown). 


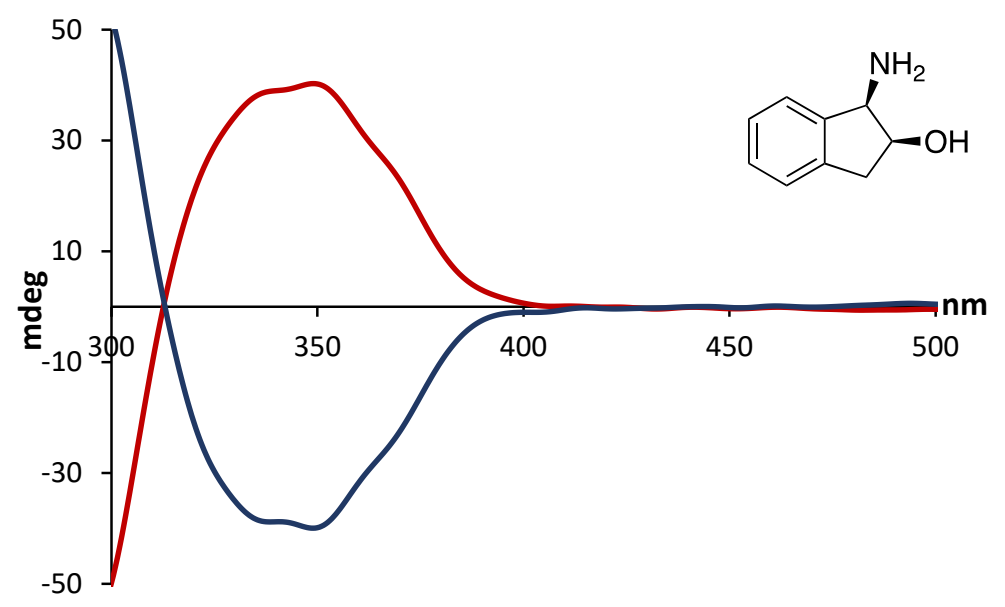

Figure S20. Sensing of $(1 R, 2 S)$-1-amino-2,3-dihydro- $1 H$-inden-2-ol (red) and (1S,2R)-1-amino2,3-dihydro- $1 \mathrm{H}$-inden-2-ol (blue) with $\mathbf{A}$ at $1.1 \mathrm{mM}$ (one enantiomeric structure shown).

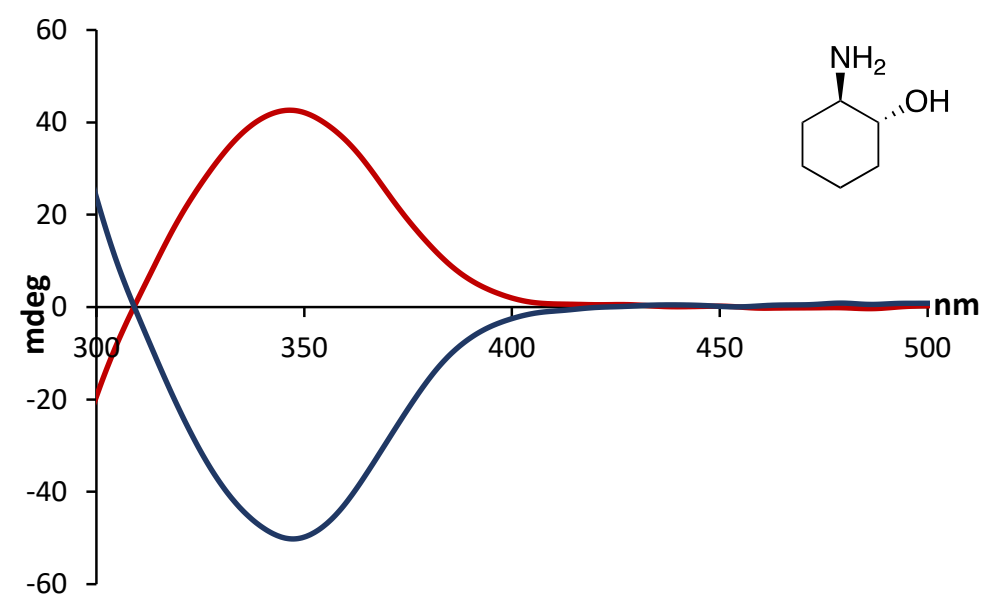

Figure S21. Sensing of (1R,2R)-2-aminocyclohexan-1-ol (red) and (1S,2S)-2-aminocyclohexan-1ol (blue) with A at $1.1 \mathrm{mM}$ (one enantiomeric structure shown). This analyte was obtained as $\mathrm{HCl}$ salt. The sensing experiments were performed in the presence of 1.0 equivalents of $\mathrm{Et}_{3} \mathrm{~N}$. 


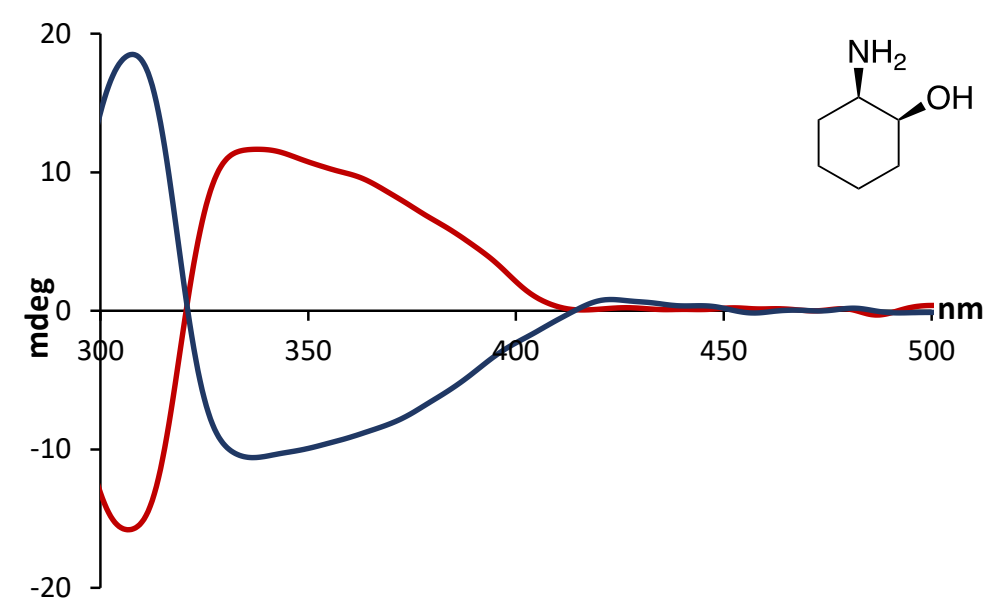

Figure S22. Sensing of (1R,2S)-2-aminocyclohexan-1-ol (red) and (1S,2R)-2-aminocyclohexan-1ol (blue) with A at $1.1 \mathrm{mM}$ (one enantiomeric structure shown). This analyte was obtained as $\mathrm{HCl}$ salt. The sensing experiments were performed in the presence of 1.0 equivalents of $\mathrm{Et}_{3} \mathrm{~N}$.

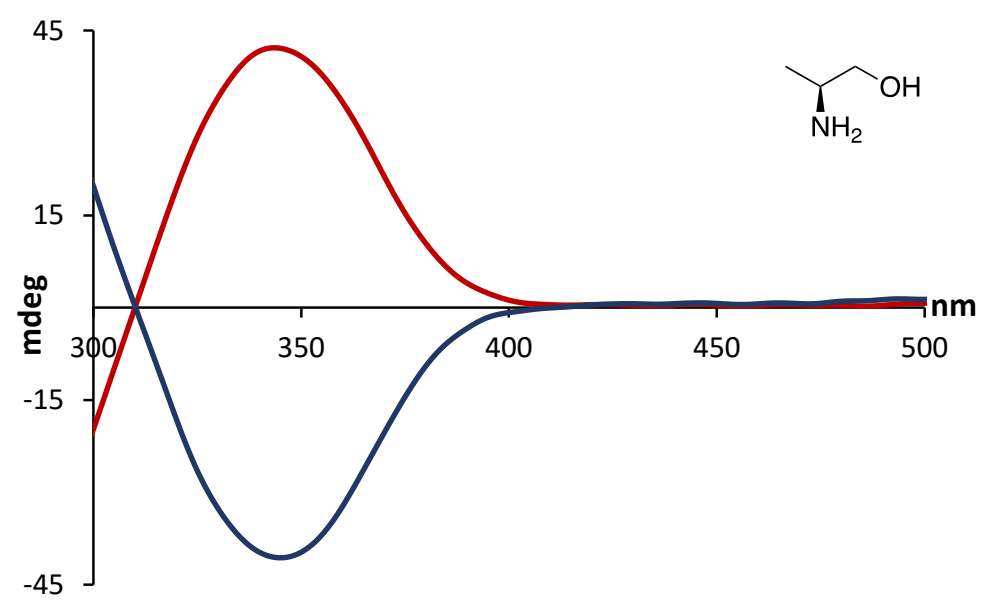

Figure S23. Sensing of $(R)$-2-aminopropan-1-ol (red) and (S)-2-aminopropan-1-ol (blue) with A at $1.1 \mathrm{mM}$. 


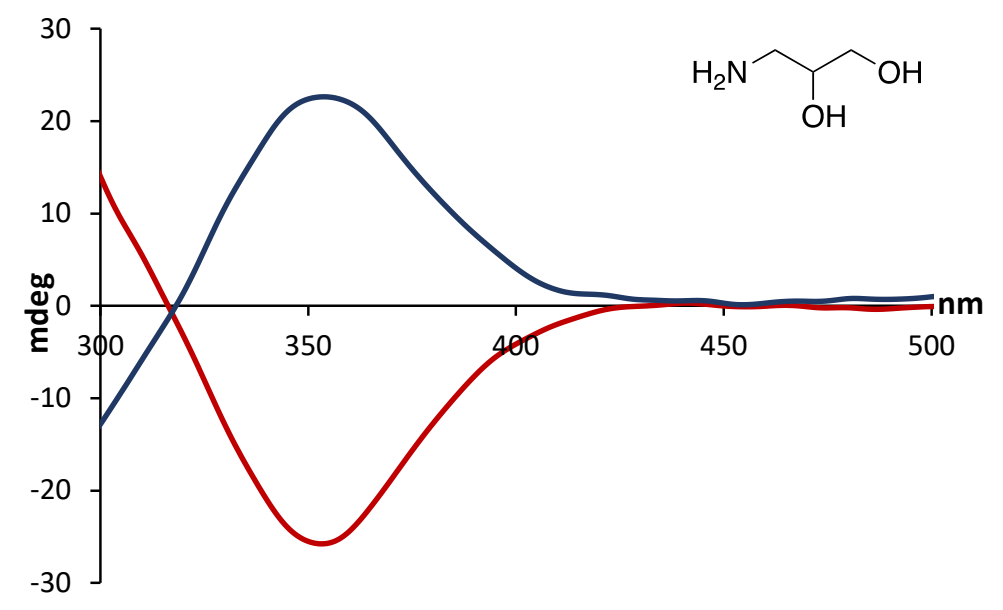

Figure S24. Sensing of ( $R$ )-3-aminopropane-1,2-diol (red) and (S)-3-aminopropane-1,2-diol (blue) with $\mathbf{A}$ at $1.1 \mathrm{mM}$.

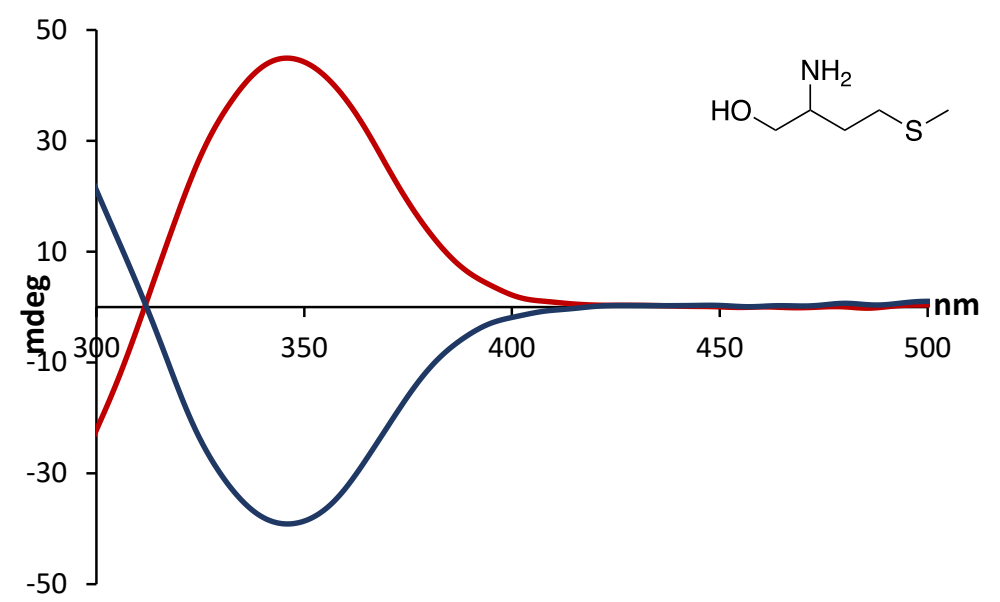

Figure S25. Sensing of (R)-2-amino-4-(methylthio)butan-1-ol (red) and (S)- 2-amino-4(methylthio)butan-1-ol (blue) with $\mathrm{A}$ at $1.1 \mathrm{mM}$.

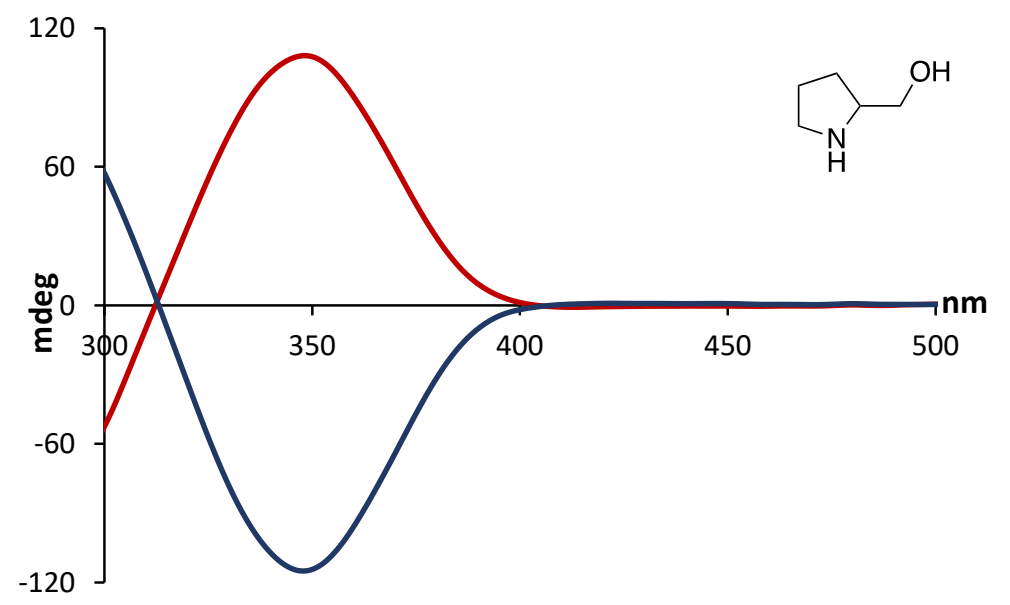

Figure S26. Sensing of $(R)$-pyrrolidin-2-ylmethanol (red) and (S)-pyrrolidin-2-ylmethanol (blue) with $A$ at $1.1 \mathrm{mM}$. 


\section{Chiroptical sensing of diamines}

Solutions containing $5.00 \mathrm{mM}$ and $10.00 \mathrm{mM}$ ninhydrin hydrate in anhydrous DMSO were prepared and used for $1.00 \mathrm{~mL}$ reactions with the chiral diamines. Solutions of the enantiopure analytes $(0.25 \mathrm{M})$ were prepared in parallel using DMSO as solvent and $10.0 \mu \mathrm{L}(0.005 \mathrm{mmol})$ were subsequently added into the reaction vials containing either one or two equivalents of $\mathbf{A}$. The samples were diluted after 20 minutes with ACN to the concentrations reported below.<smiles>N[C@@H]1CCCC[C@H]1N</smiles><smiles>N[C@@H](c1ccccc1)[C@H](N)c1ccccc1</smiles>

Figure S27. Structures of diamines tested.

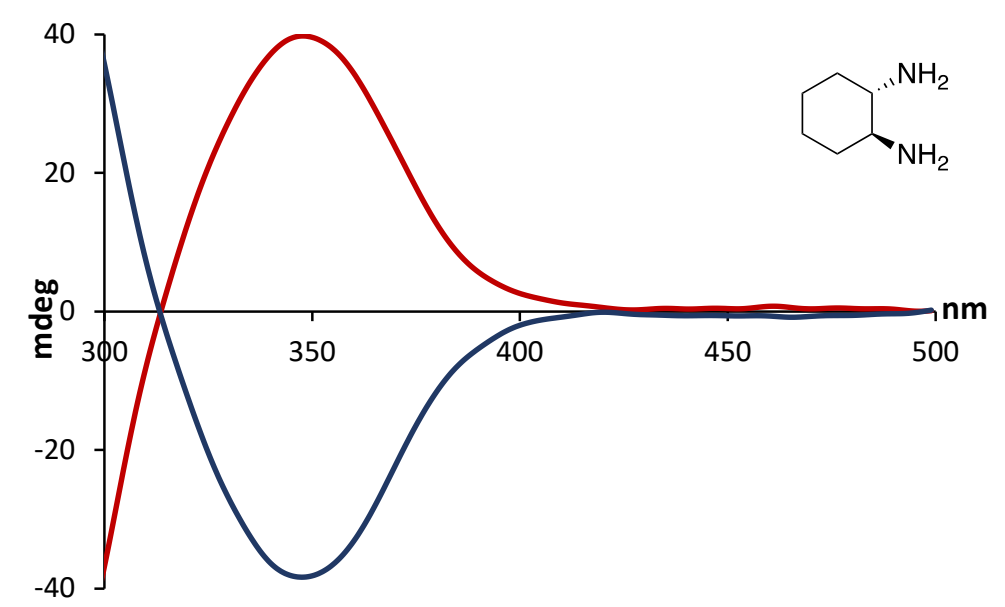

Figure S28. Sensing of $(1 R, 2 R)$-diaminocyclohexane (red) and $(1 S, 2 S)$-diaminocyclohexane (blue) with $A$ at $1.1 \mathrm{mM}$.

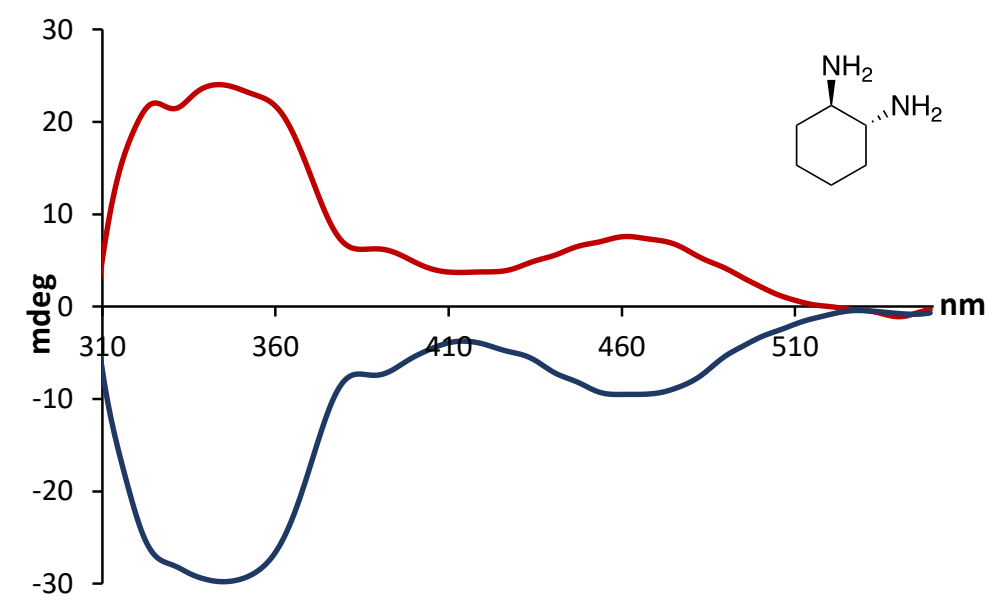

Figure S29. Sensing of $(1 R, 2 R)$-diaminocyclohexane (red) and (1S,2S)-diaminocyclohexane (blue) with 2 equivalents of $A$ at $1.65 \mathrm{mM}$. 


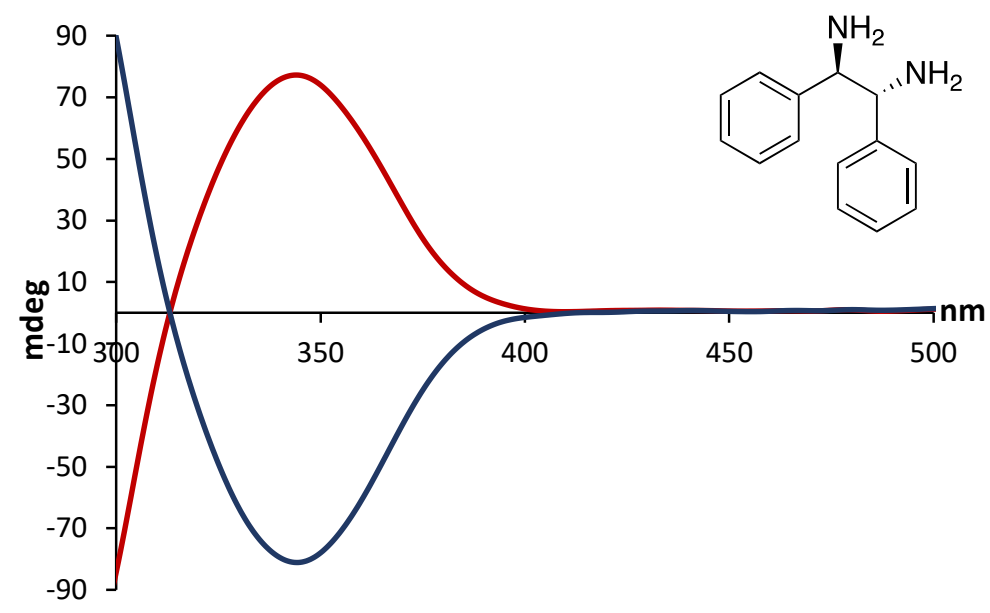

Figure S30. CD spectra of the analyte-bound species formed from ninhydrin-hydrate $A$ with $(1 R, 2 R)$-diphenylethane-1,2-diamine (red) and (1S,2S)-diphenylethane-1,2-diamine (blue) at 1.1 $\mathrm{mM}$.

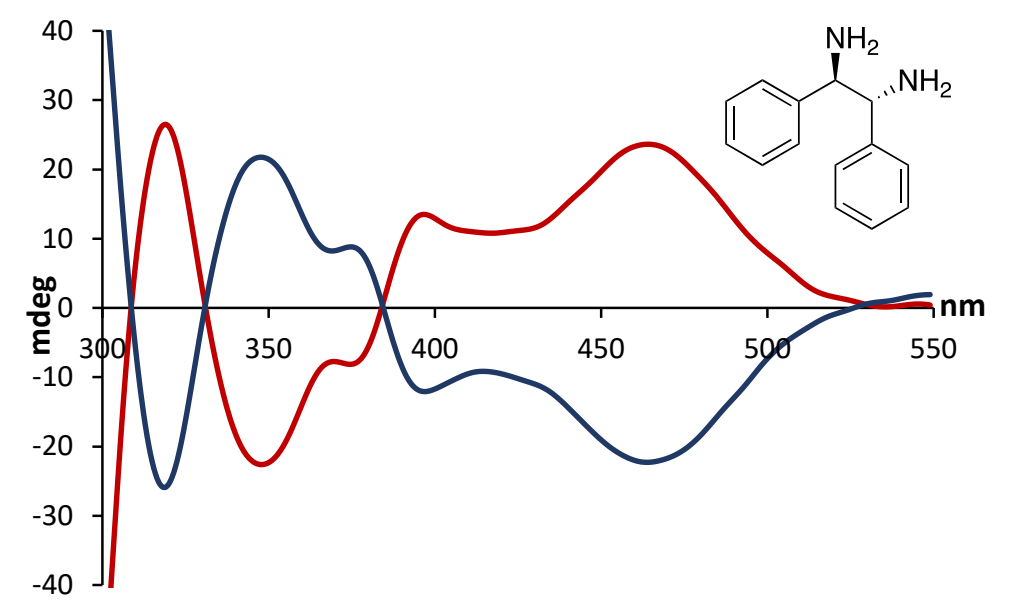

Figure S31. Sensing of $(1 R, 2 R)$-diphenylethane-1,2-diamine (red) and $(1 S, 2 S)$-diphenylethane1,2-diamine (blue) with 2 equivalents of $A$ at $1.1 \mathrm{mM}$. 


\section{Optimization and mechanistic studies}

\subsection{NMR analysis}

A solution of ninhydrin hydrate in DMSO- $d^{6}(18.7 \mathrm{mM})$ was prepared and ${ }^{1} \mathrm{H}$ NMR experiments were conducted with varied amounts of $(R)$-phenylethan-1-amine $(0.0,0.5,1.0,1.5,2.0$ molar equivalents) present.
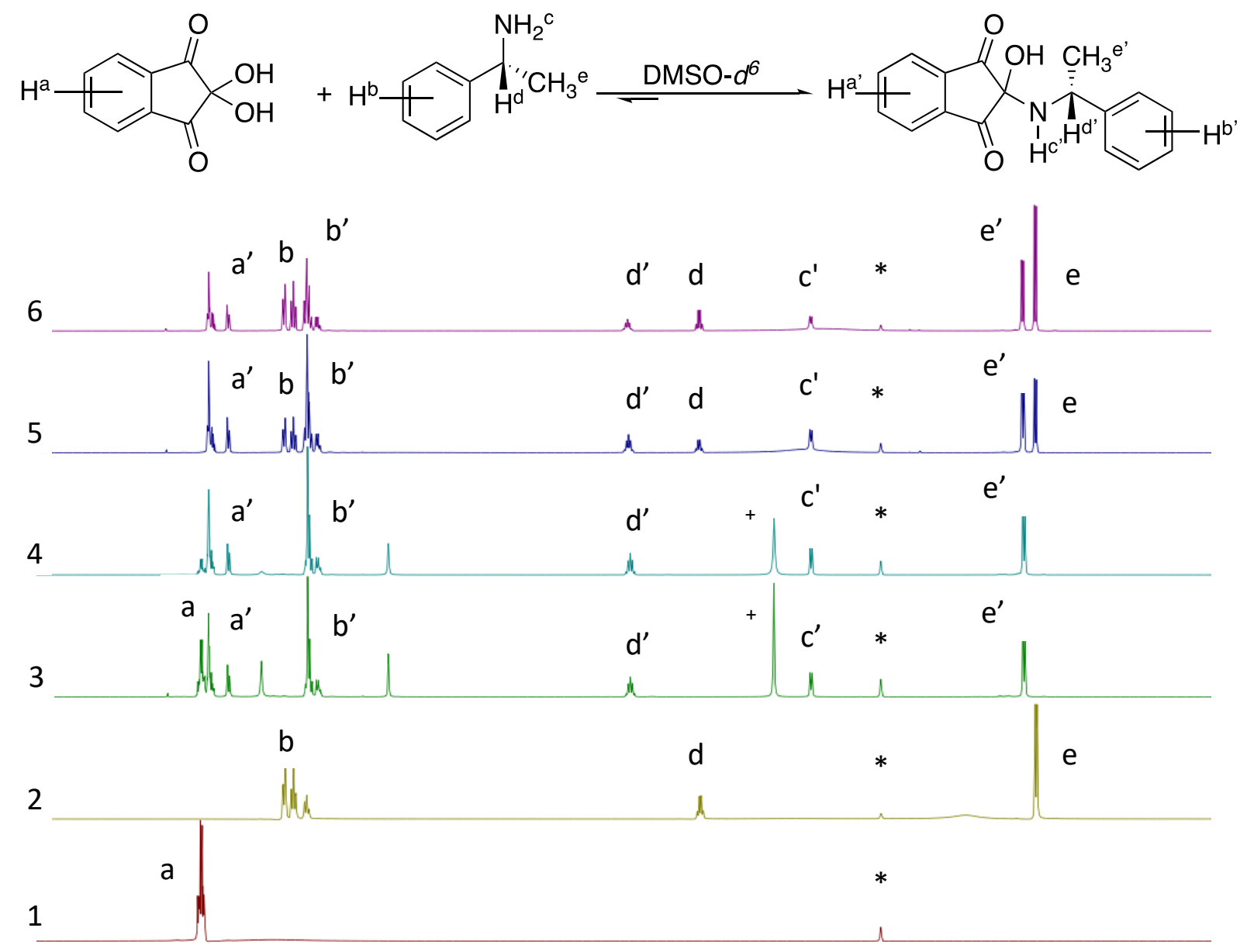

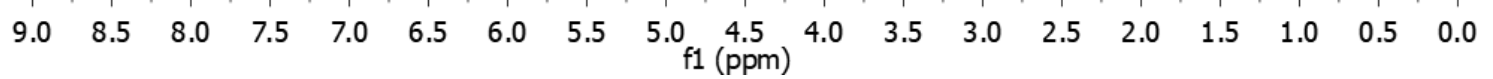

Figure S32. Titration experiment with phenylethylamine, 1, and A. From bottom spectra to top:

(1) ninhydrin hydrate, (2) phenylethan-1-amine, (3-6) ninhydrin hydrate with 0.5 (3), 1.0 (4), 1.5 (5), 2.0 (6) equivalents of phenylethan-1-amine. ${ }^{*}$ DMSO solvent signal; ${ }^{+} \mathrm{H}_{2} \mathrm{O}$. 
A solution of ninhydrin hydrate in DMSO- $d^{6}(18.7 \mathrm{mM})$ was prepared with one stoichiometric equivalent of $(R)$-phenylethan-1-amine and analyzed by ${ }^{1} \mathrm{H}$ NMR spectroscopy (red bottom spectrum). $\mathrm{D}_{2} \mathrm{O}$ ( 0.5 molar equivalents) was then added to the reaction (blue-green top spectrum). The results show apparent peak broadening of the signal at $6.48 \mathrm{ppm}$ indicating the presence of an exchangeable proton (X). Cleavage of the acetal upon $\mathrm{D}_{2} \mathrm{O}$ addition is evident from the development of the free amine proton signals $\left({ }^{*}\right)$. Taken together, the spectra indicate a reversible and dynamic covalent process in solution.
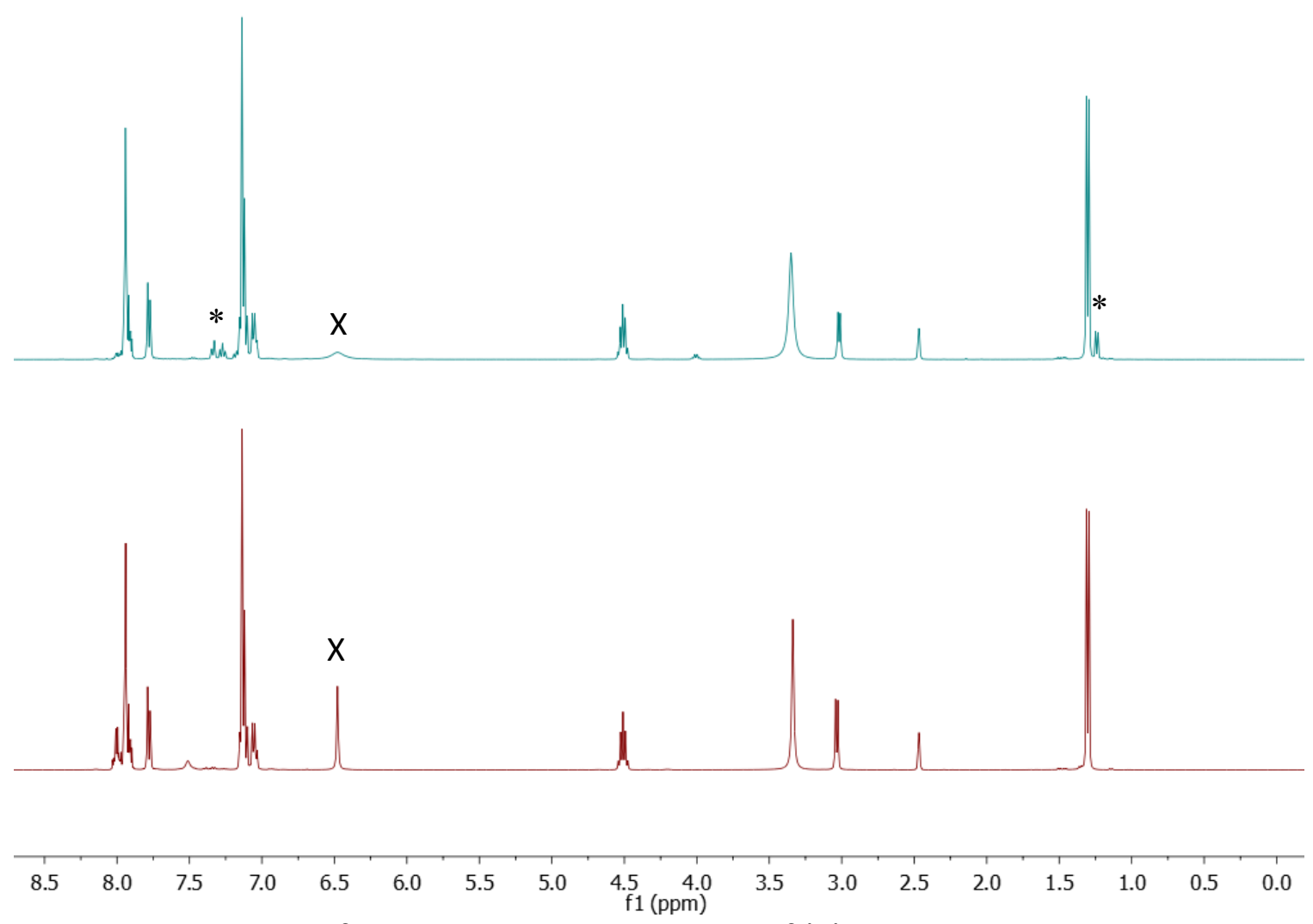

Figure S33. Addition of $\mathrm{D}_{2} \mathrm{O}$ into a reaction mixture of $(R)$-phenylethan-1-amine and $\mathbf{A}$. 
The following NMR studies were conducted with $10.0 \mathrm{mM} \mathrm{DMSO}-d^{6}$ solutions containing the analyte and ninhydrin in equimolar amounts. The solutions were reacted for 15 minutes prior to the analysis.
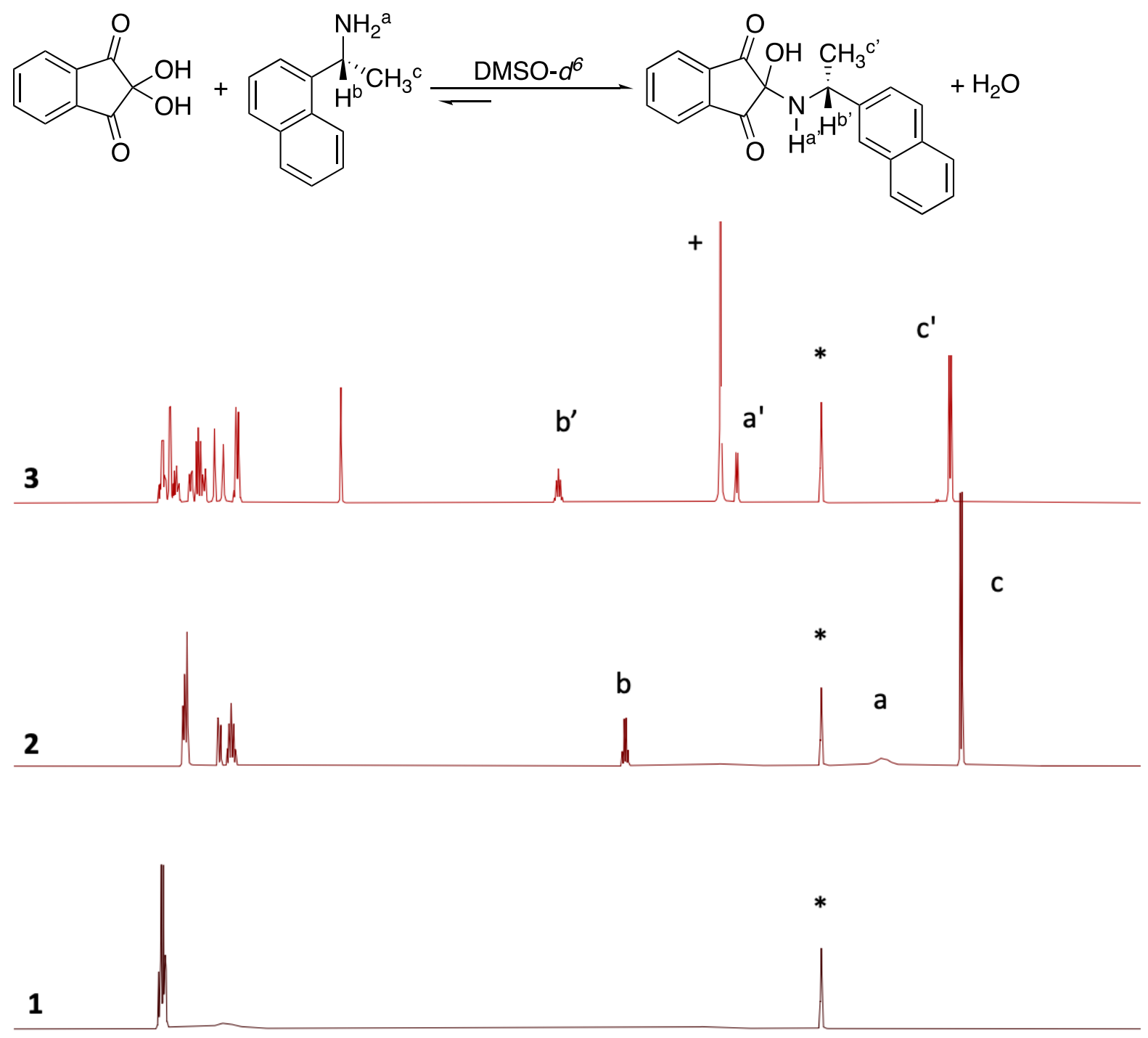

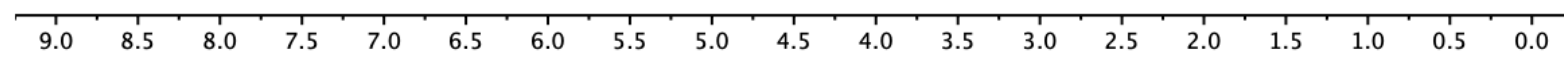

Figure S34. NMR analysis of the reaction between analyte 6 amd A. From bottom spectra to top: (1) ninhydrin hydrate, (2) (R)-1-(naphthalen-2-yl)ethan-1-amine, (3) ninhydrin hydrate with 1.0 equivalent of $(R)$-1-(naphthalen-2-yl)ethan-1-amine. ${ }^{*}$ DMSO solvent signal; ${ }^{+} \mathrm{H}_{2} \mathrm{O}$. 
<smiles>CC(C)C(C)(N)C(C)(C)[PH3+]</smiles>
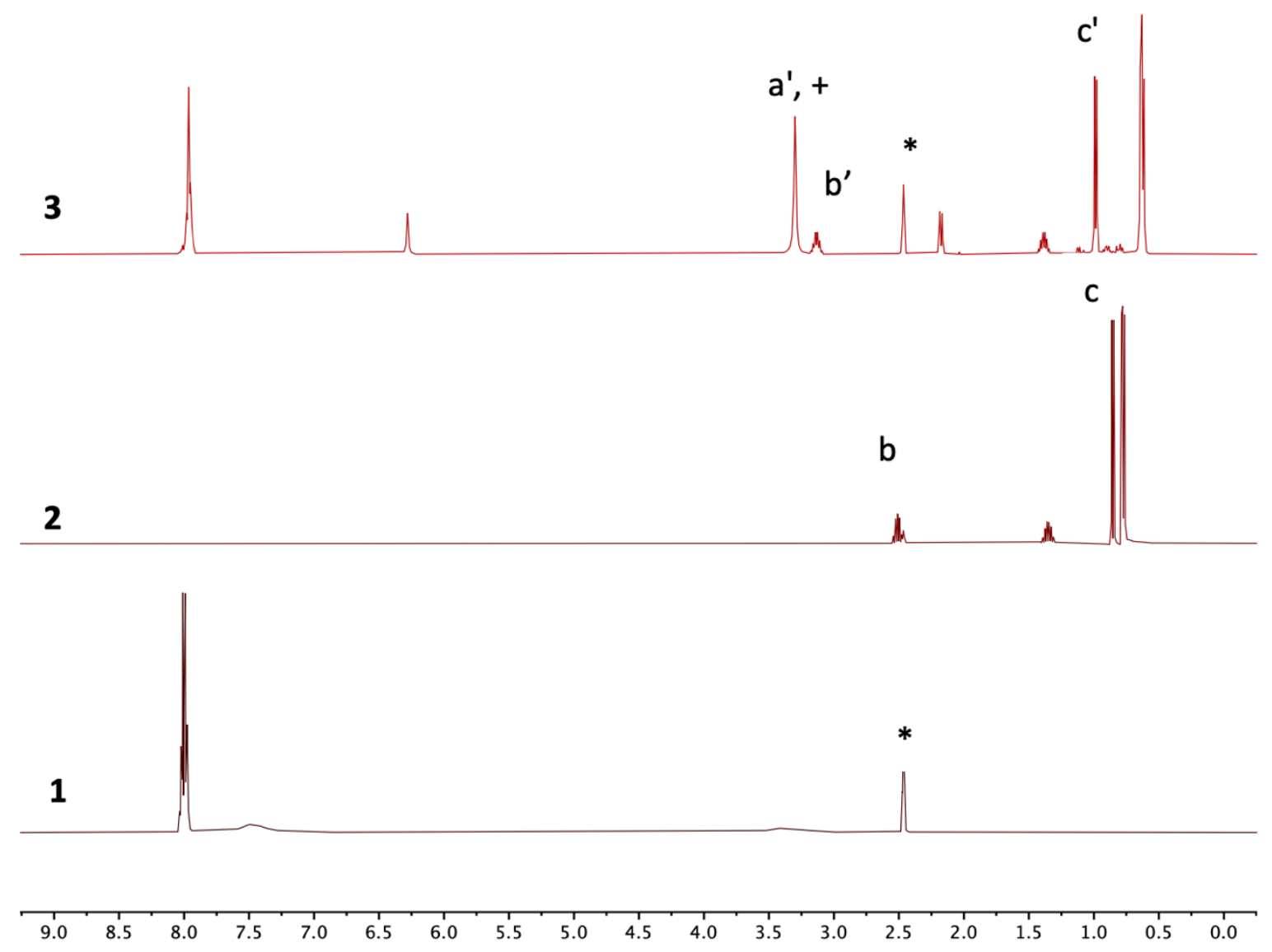

Figure S35. NMR analysis of the reaction between analyte $\mathbf{7}$ and A. From bottom spectra to top: (1) ninhydrin hydrate, (2) (R)-3-methylbutan-2-amine, (3) ninhydrin hydrate with 1.0 equivalent of $(R)$-3-methylbutan-2-amine. ${ }^{*}$ DMSO solvent signal; ${ }^{+} \mathrm{H}_{2} \mathrm{O}$. 

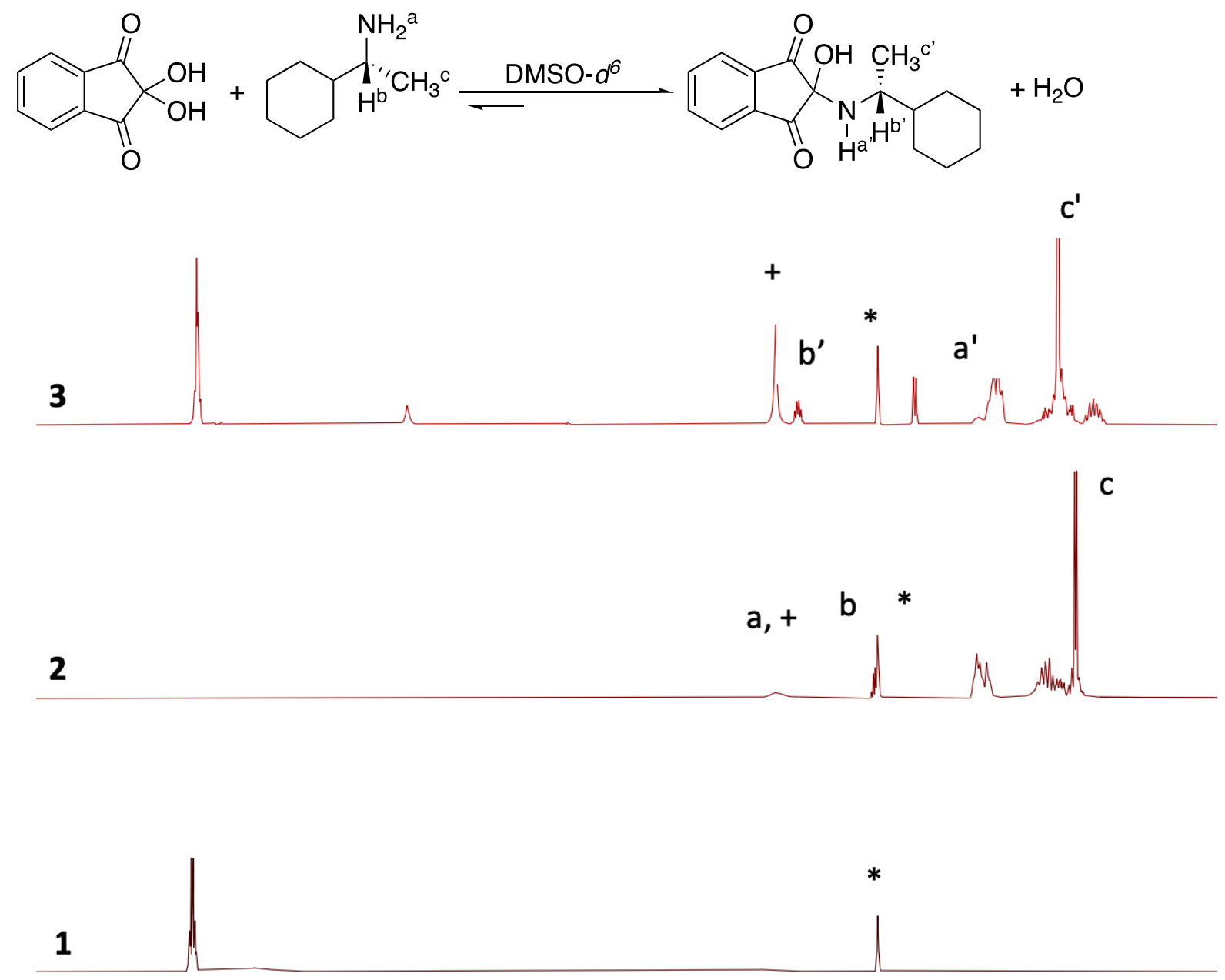

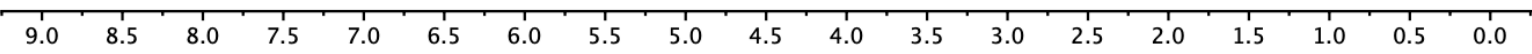

Figure S36. NMR analysis of the reaction between analyte 9 and A. From bottom spectra to top: (1) ninhydrin hydrate, (2) (R)-1-cyclohexylethan-1-amine, (3) ninhydrin hydrate with 1.0 equivalent of (S)-1-cyclohexylethan-1-amine. *DMSO solvent signal; ${ }^{+} \mathrm{H}_{2} \mathrm{O}$. 

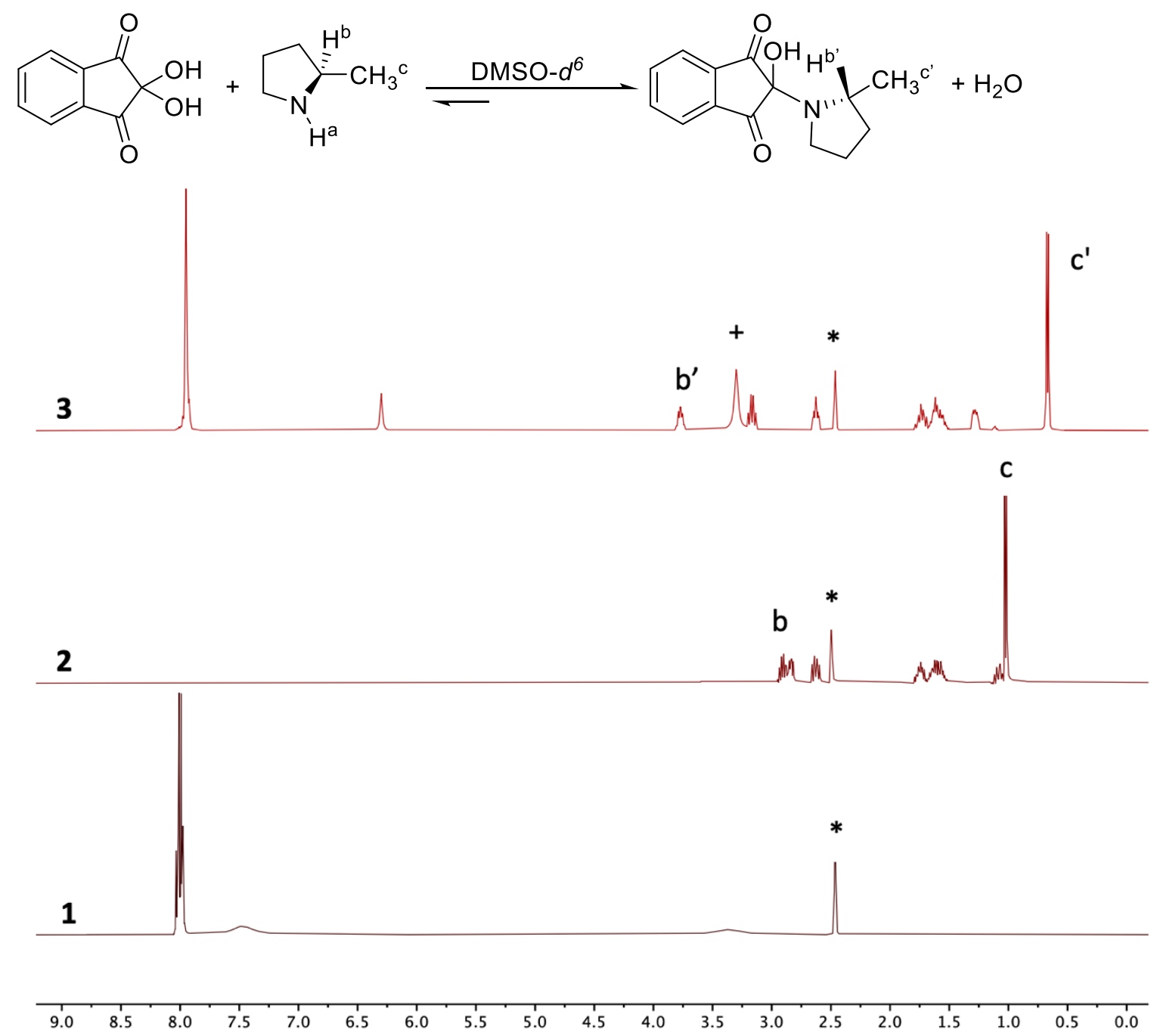

Figure S37. NMR analysis of the reaction between analyte $\mathbf{1 0}$ and $\mathbf{A}$. From bottom spectra (red) to top: (1) ninhydrin hydrate, (2) (R)-2-methylpyrrolidine, (3) ninhydrin hydrate with 1.0 equivalent of $(R)$-2-methylpyrrolidine. ${ }^{*}$ DMSO solvent signal; ${ }^{+} \mathrm{H}_{2} \mathrm{O}$. 
<smiles>CO[C@H](C)[C@H](N)CO</smiles>

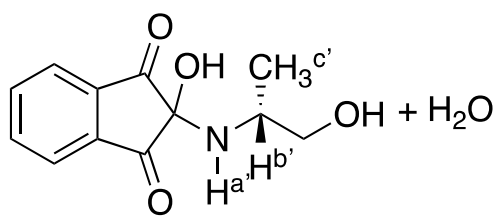

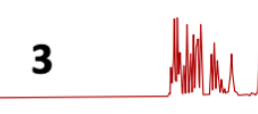

$a^{\prime},+$

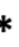

2

b

C
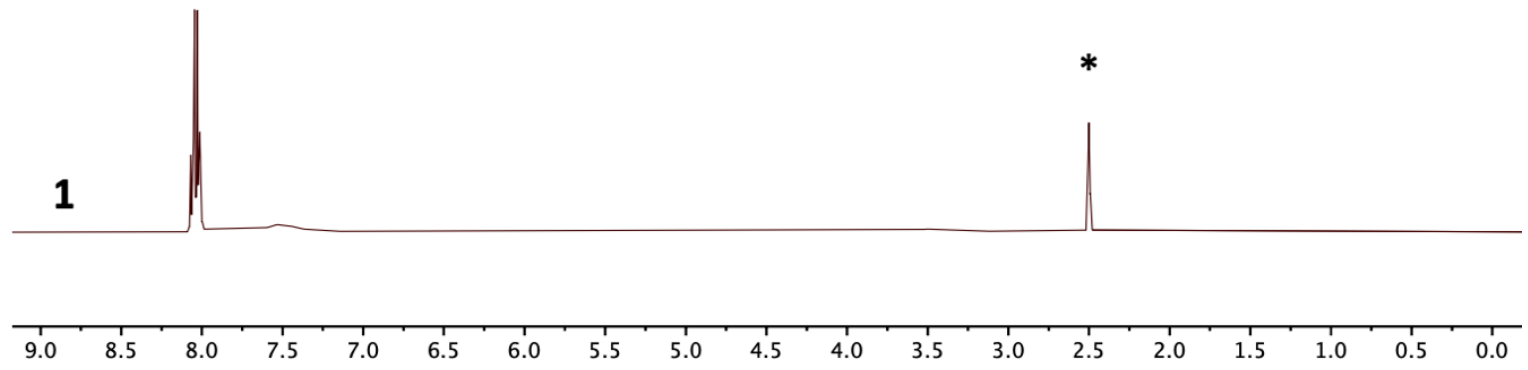

Figure S38. NMR analysis of the reaction between analyse $\mathbf{1 9}$ and $\mathbf{A}$. From bottom spectra to top: (1) ninhydrin hydrate, (2) (R)-2-aminopropan-1-ol, (3) ninhydrin hydrate with 1.0 equivalent of $(R)$-2-aminopropan-1-ol. *DMSO solvent signal; ${ }^{+} \mathrm{H}_{2} \mathrm{O}$.

S21 


\subsection{UV analysis}

UV-vis spectroscopy was conducted with a series of analytes using stoichiometric amounts of ninhydrin hydrate (blue) following the protocol described above for CD sensing. For comparison, the UV-vis spectrum of ninhydrate hydrate in solution (red) is shown. For UV-vis analysis, the $5.0 \mathrm{mM}$ DMSO reactions were diluted to $0.600 \mathrm{mM}$ with acetonitrile. In all cases, the ninhydrin adducts show increased absorbance above $270 \mathrm{~nm}$.

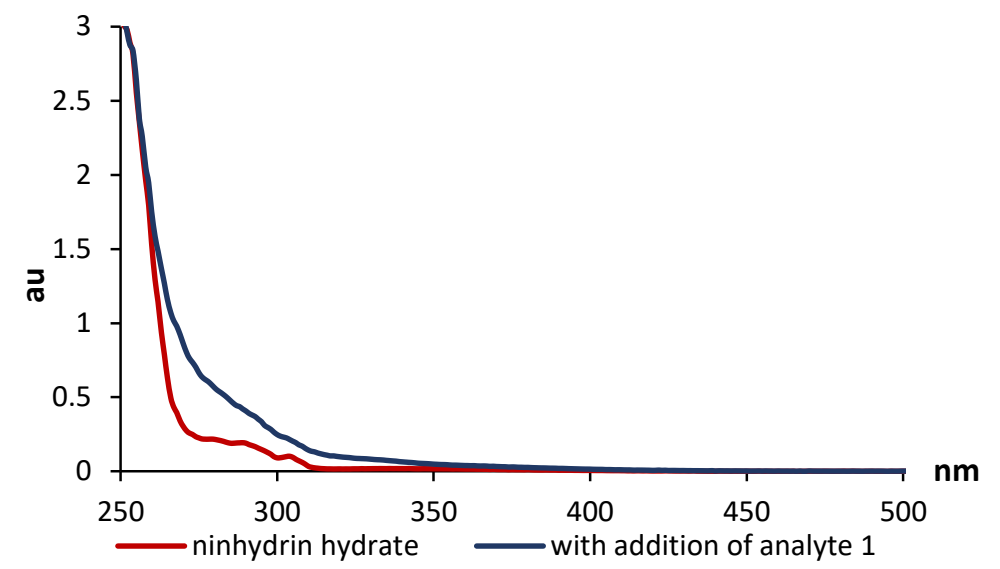

Figure S39. UV spectra of $\mathbf{A}$ and the reaction mixture with 1.

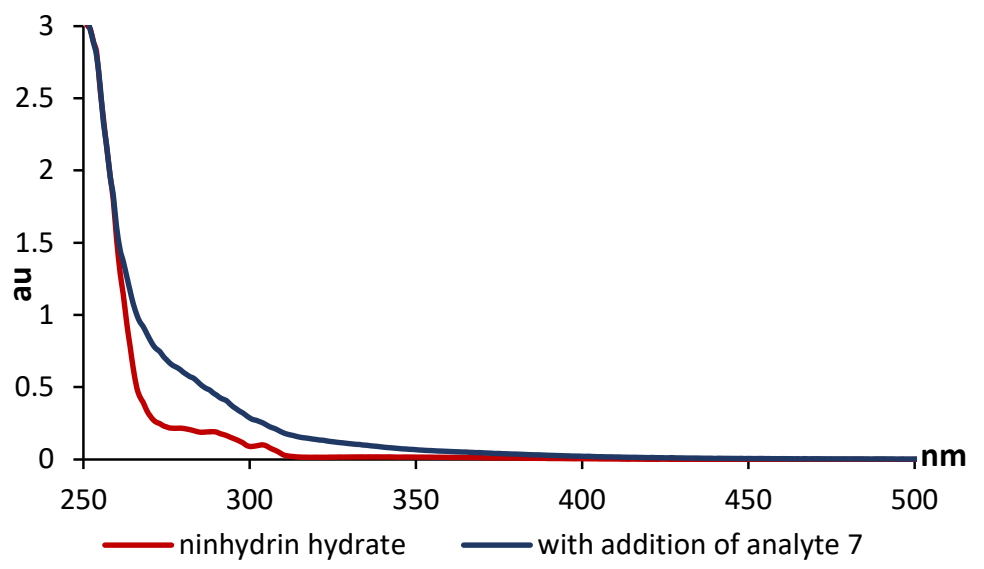

Figure S40. UV spectra of $\mathbf{A}$ and the reaction mixture with $\mathbf{7 .}$ 


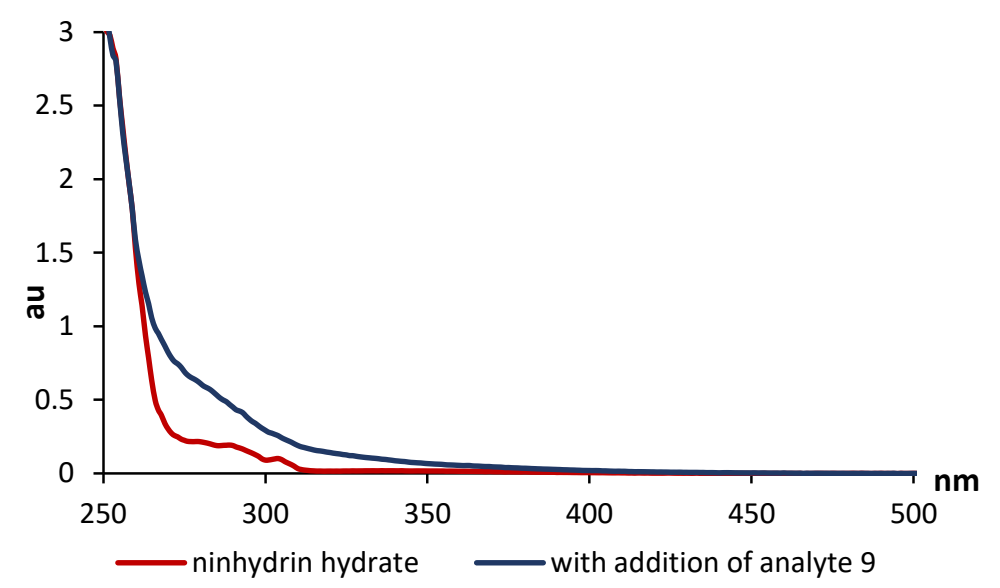

Figure S41. UV spectra of A and the reaction mixture with 9.

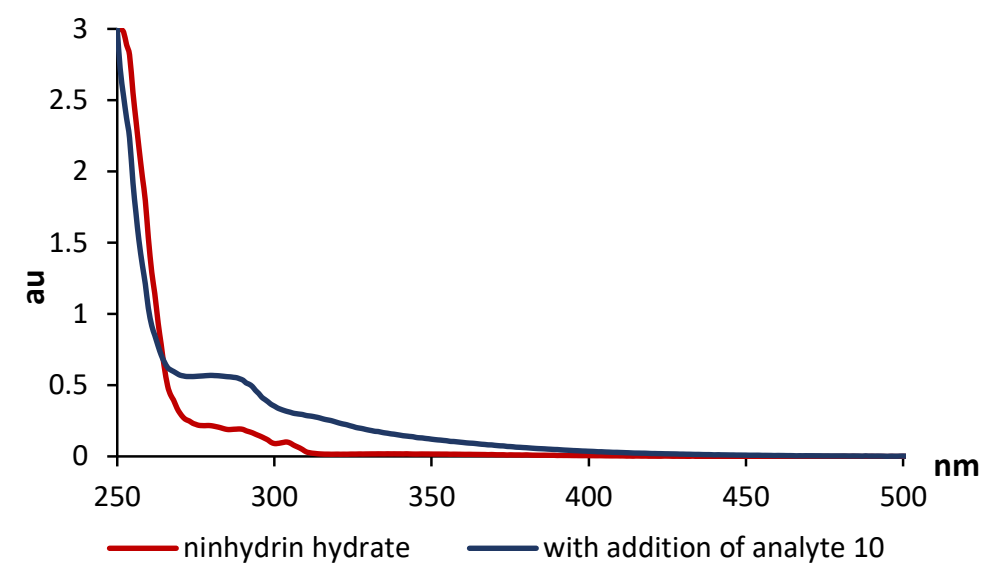

Figure S42. UV spectra of $\mathbf{A}$ and the reaction mixture with $\mathbf{1 0 .}$

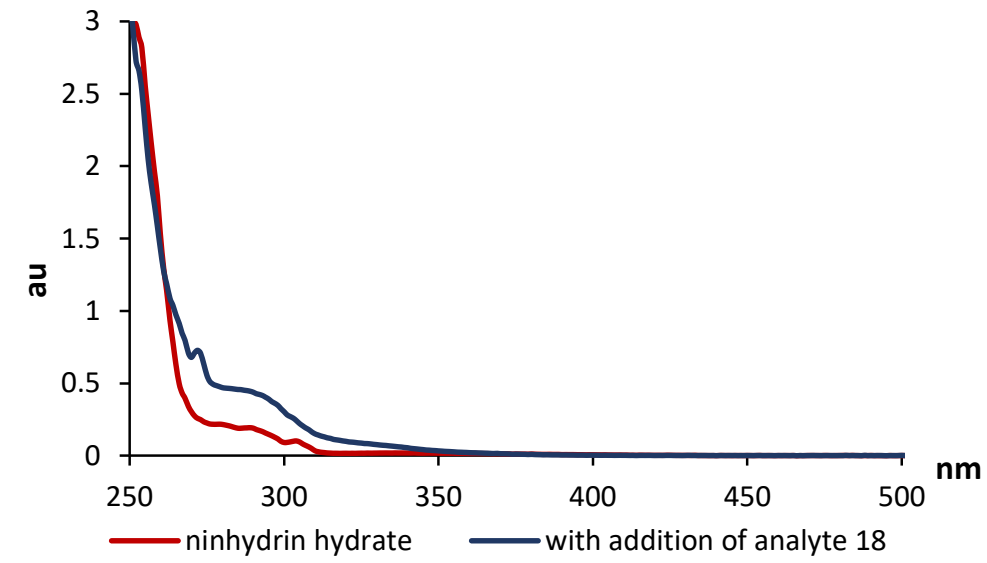

Figure S43. UV spectra of $\mathbf{A}$ and the reaction mixture with $\mathbf{1 8 .}$ 


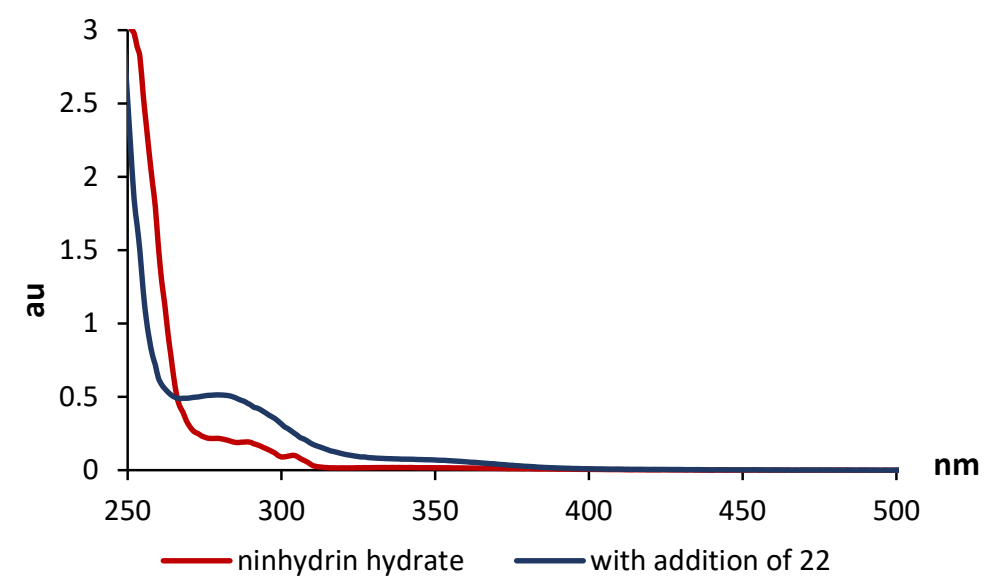

Figure S44. UV spectra of A and the reaction mixture with 22. 


\subsection{CD analysis confirming stoichiometric binding}

$\mathrm{CD}$ spectra of the adduct formed from ninhydrin hydrate and one equivalent of $(R)$-1-

phenylethan-1-amine (red) or 2 equivalents of $(R)$-1-phenylethan-1-amine (blue) in DMSO at 5.0 $\mathrm{mM}$. CD analysis was performed after dilution with ACN to $1.1 \mathrm{mM}$. The results are in agreement with 1:1 stoichiometric binding.

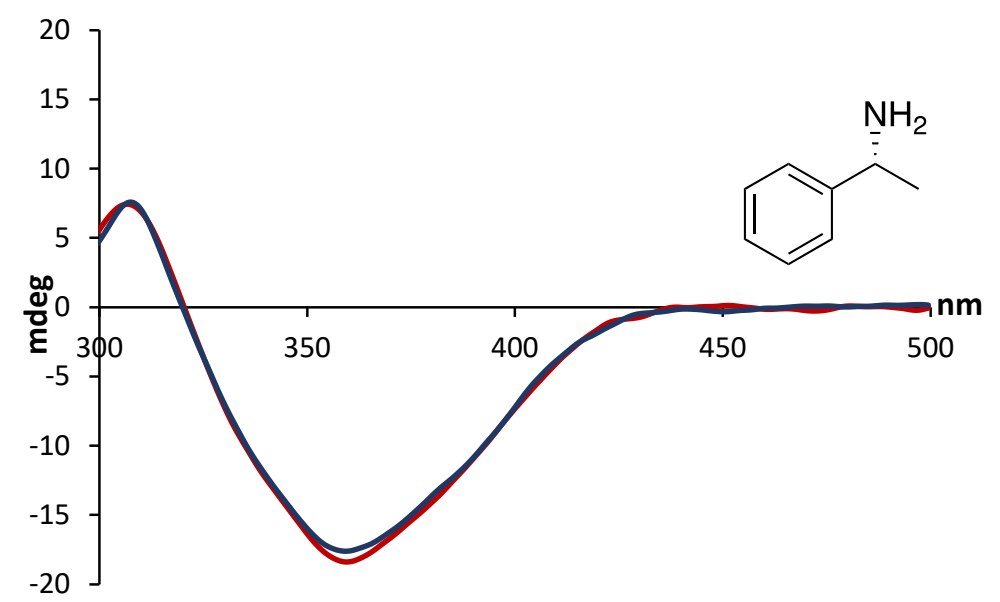

Figure S45. CD sensing using A with varied amounts of $\mathbf{1}$.

CD spectra of the adduct formed from ninhydrin hydrate and varied equivalents of $(R)-2$ methylpyrrolidine in DMSO at 5.0 mM (0.5-grey; 1.0-black; 1.5-red; 2.0-light blue; 4.0-yellow series. CD analysis was performed after dilution with ACN to $1.1 \mathrm{mM}$.

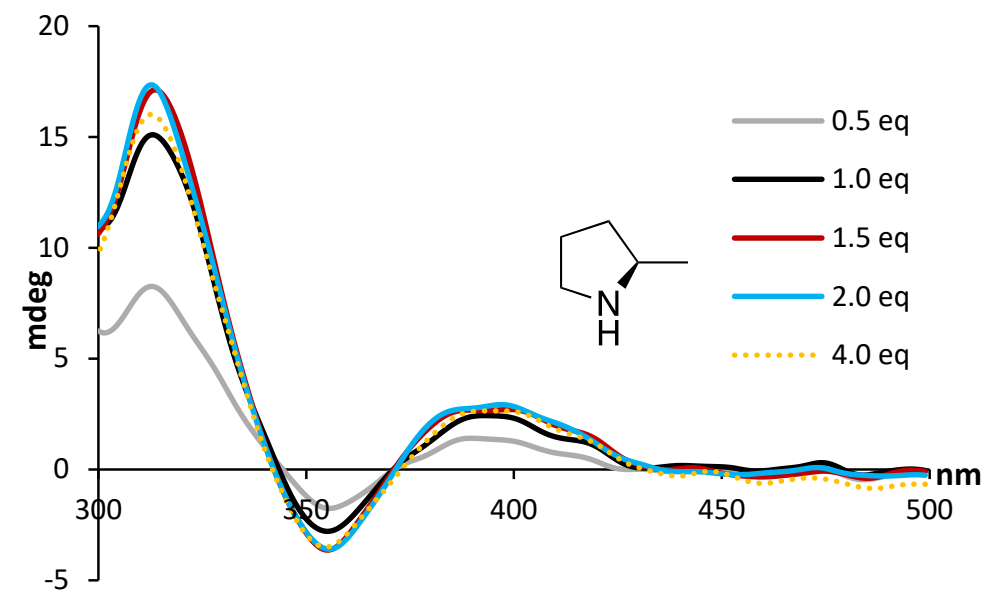

Figure S46. CD sensing using A with varied amounts of 10. 


\subsection{Reaction time and product stability}

Solutions were prepared according to the $C D$ sensing protocol described above. The acetal formed from $(R)$-1-phenylethan-1-amine and ninhydrin was subsequently subjected to $C D$ measurements (at $1.1 \mathrm{mM}$ ) for a total time of 120 minutes. The reaction is completed within 10 minutes when the maximum CD was observed.
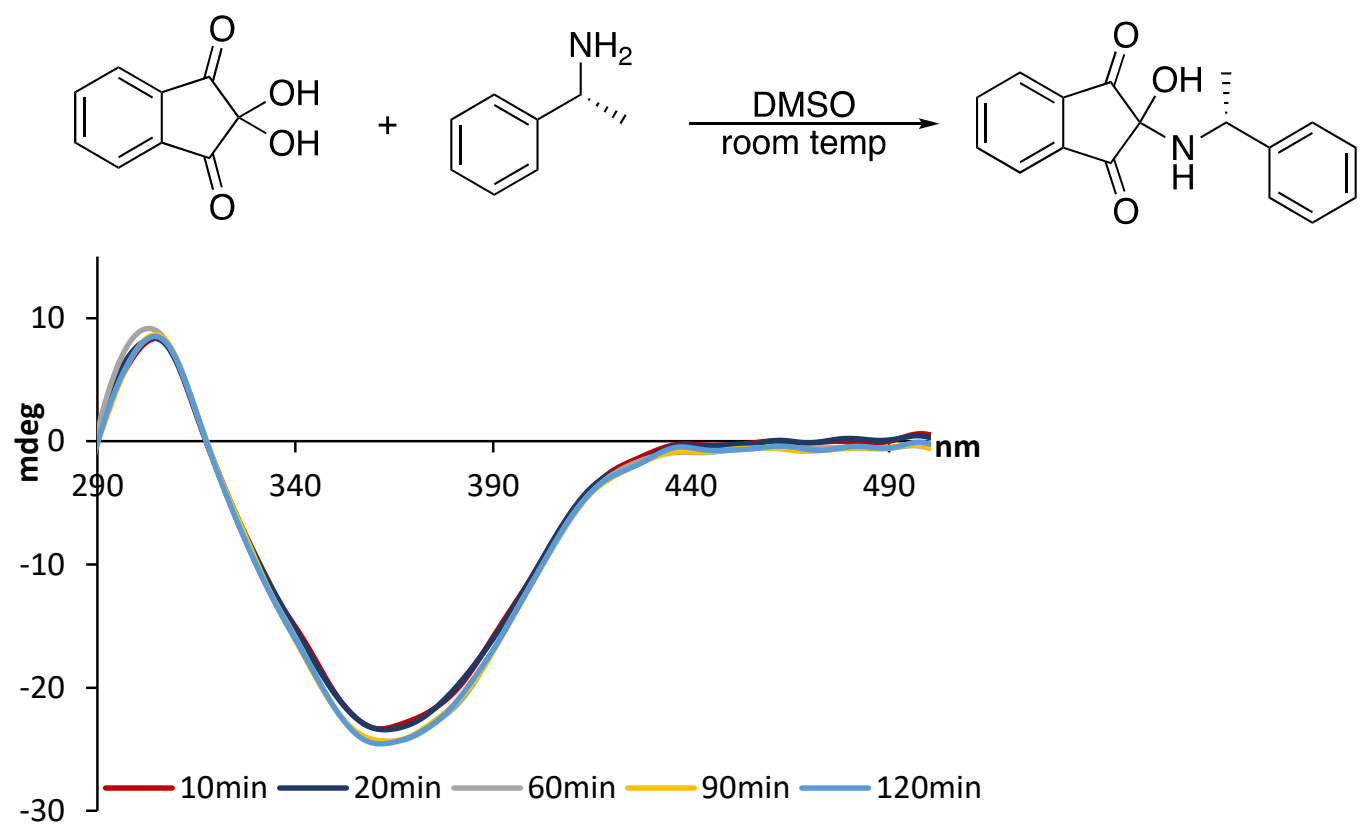

Figure S47. CD sensing of 1 over time.

Solutions were prepared according to the CD sensing protocol described above. The species formed from $(R)$-1-phenylpropan-1-amine and ninhydrin was subsequently subjected to $C D$ measurements (at $1.1 \mathrm{mM}$ ) for a total time of 20 minutes. The reaction is completed within 10 minutes when the maximum CD was observed.

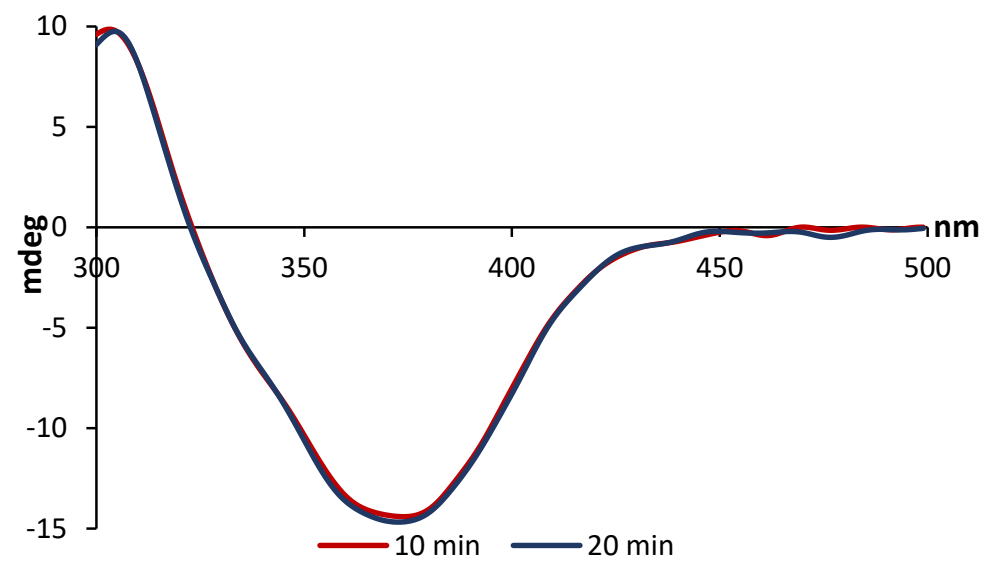

Figure S48. CD sensing of 2 over time. 
Solutions were prepared according to the CD sensing protocol described above. The species formed from $(R)$-1-(pyridin-3-yl)ethan-1-amine and ninhydrin was subsequently subjected to CD measurements (at $1.1 \mathrm{mM}$ ) for a total time of 20 minutes. The reaction is completed within 10 minutes when the maximum CD was observed.

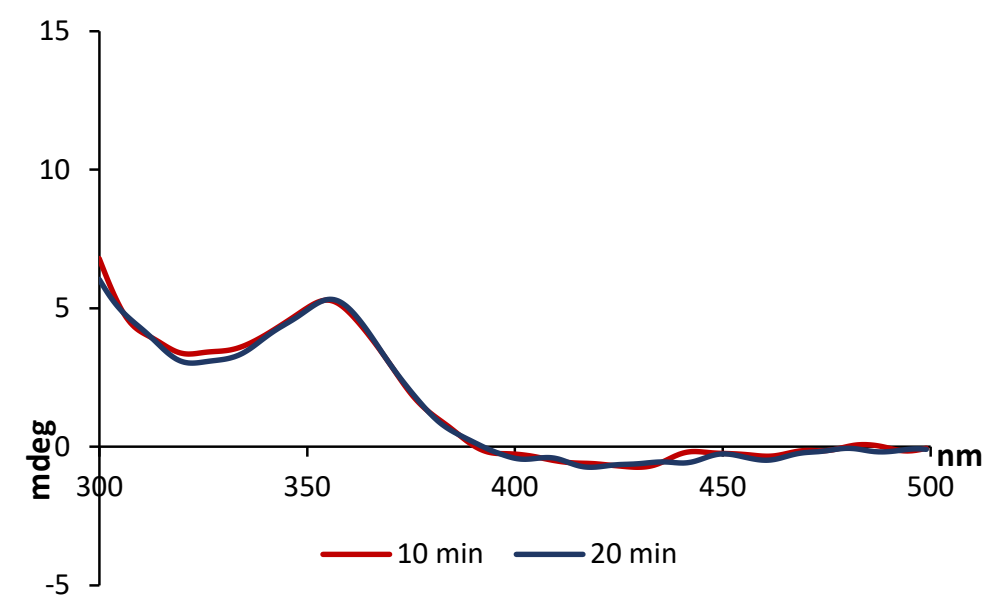

Figure S49. CD sensing of 4 over time.

Solutions were prepared according to the CD sensing protocol described above. The species formed from $(R)$-1-(naphthalen-2-yl)ethan-1-amine and ninhydrin was subsequently subjected to $\mathrm{CD}$ measurements (at $1.1 \mathrm{mM}$ ) for a total time of 30 minutes. The reaction is completed within 10 minutes when the maximum CD was observed.

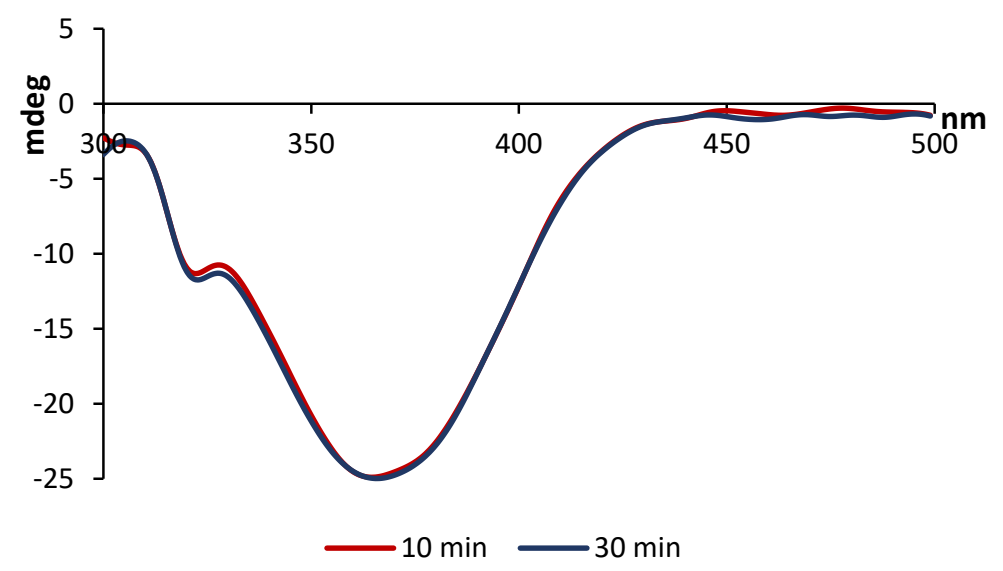

Figure S50. CD sensing of 6 over time. 
Solutions were prepared according to the CD sensing protocol described above. The species formed from $(R)$-1-cyclohexylethan-1-amine and ninhydrin was subsequently subjected to CD measurements (at $1.1 \mathrm{mM}$ ) for a total time of 20 minutes. The reaction is completed within 10 minutes when the maximum CD was observed.

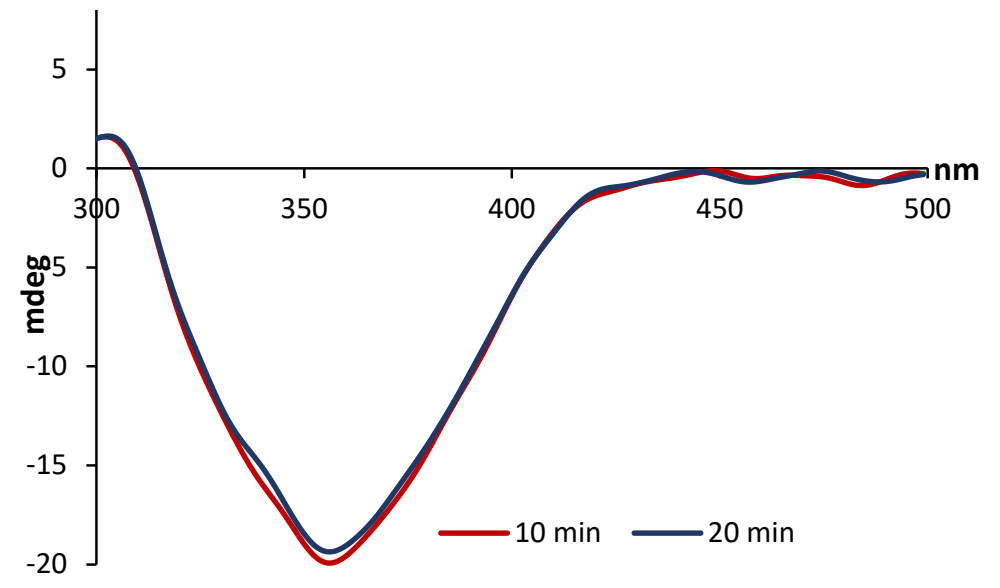

Figure S51. CD sensing of 9 over time.

Solutions were prepared according to the CD sensing protocol described above. The species formed from $(R)$-methylpyrrolidine and ninhydrin was subsequently subjected to $C D$ measurements (at $1.1 \mathrm{mM}$ ) for a total time of 45 minutes. The reaction is completed within 10 minutes when the maximum CD was observed.

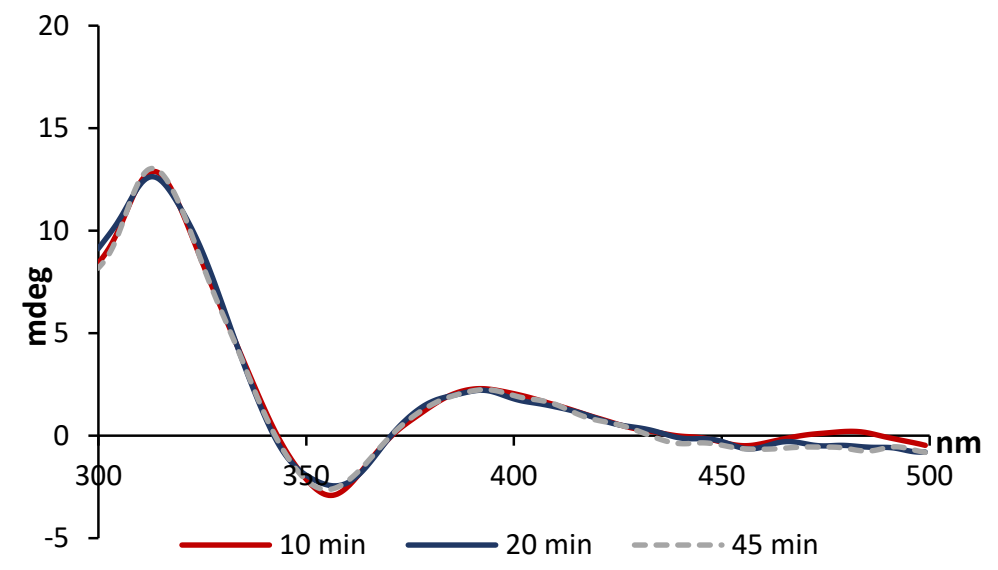

Figure S52. CD sensing of $\mathbf{1 0}$ over time. 
Solutions were prepared according to the $C D$ sensing protocol described above. The species formed from $(R)$-2-amino-2-phenylethan-1-ol and ninhydrin was subsequently subjected to $C D$ measurements (at $1.1 \mathrm{mM}$ ) for a total time of 20 minutes. The reaction is completed within 10 minutes when the maximum CD was observed.

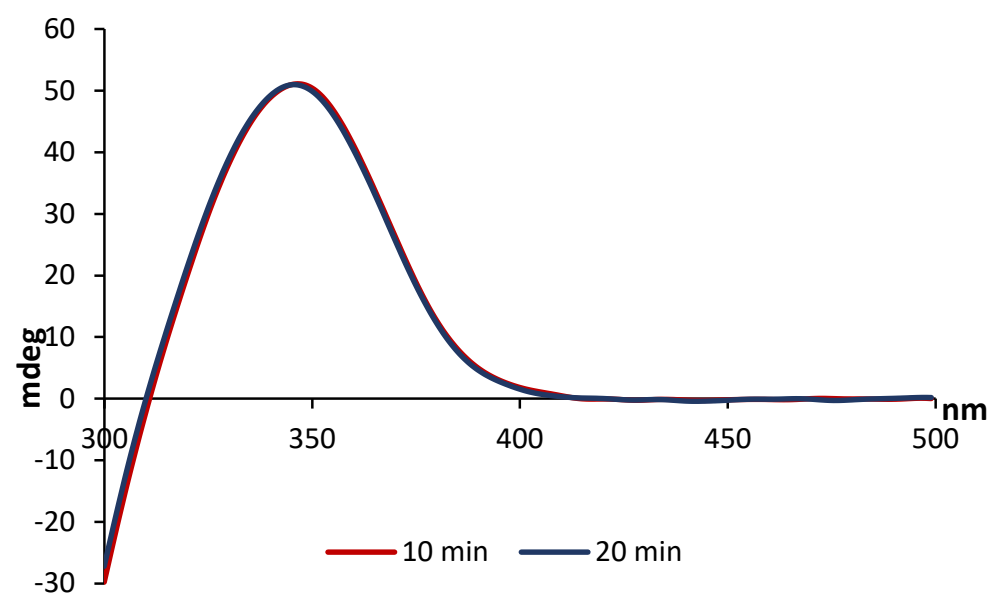

Figure S53. CD sensing of 11 over time.

Solutions were prepared according to the CD sensing protocol described above. The species formed from $(R)$-3-aminopropane-1,2-diol and ninhydrin was subsequently subjected to $C D$ measurements (at $1.1 \mathrm{mM}$ ) for a total time of 35 minutes. The reaction is almost completed after 10 minutes.

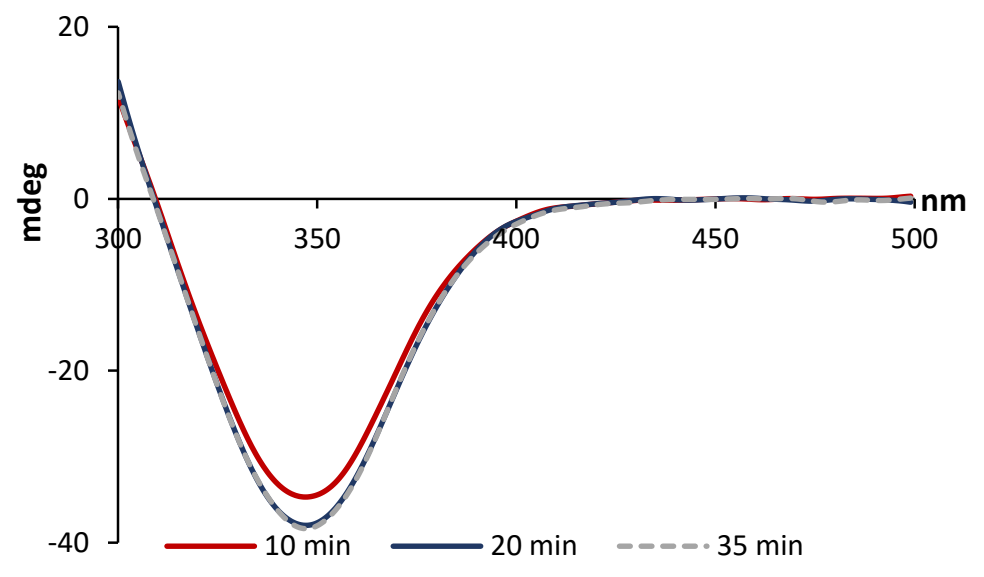

Figure S54. CD sensing of 20 over time. 


\subsection{Sensing solvent selection}

Solutions were prepared according to the above CD sensing protocol. A $5.00 \mathrm{mM}$ solution of the ninhydrin adduct of $(R)$-phenylethan-1-amine was prepared in DMSO. A portion of the reaction mixture was diluted to $1.1 \mathrm{mM}$ resulting in the bulk sensing solvent as either ACN (red), dichloromethane (dark blue), THF (grey), DMSO (light blue) or $\mathrm{MeOH}$ (black) and subsequently subjected to $C D$ analysis.

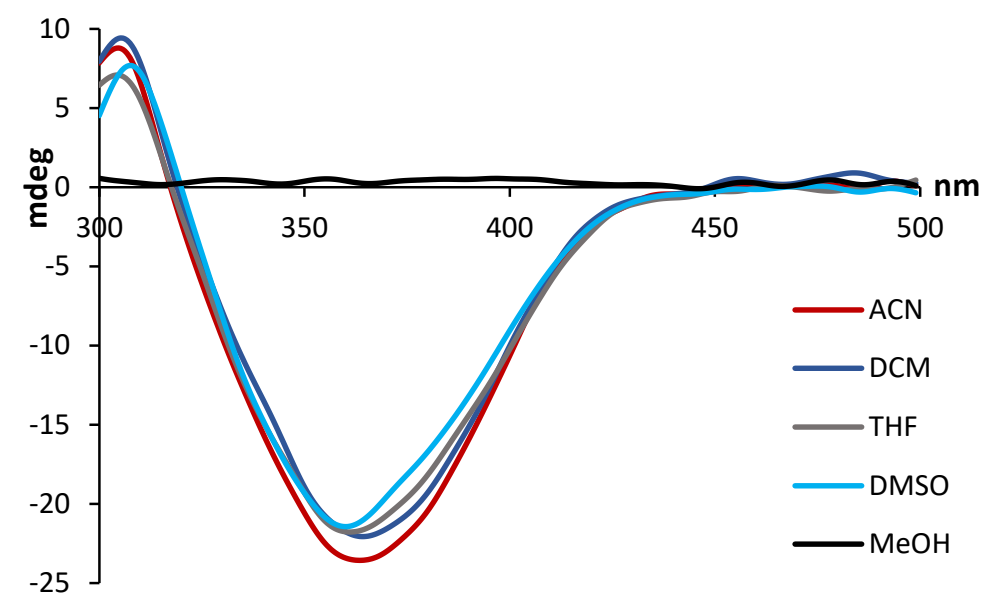

Figure S55. CD analysis of 1A with various diluting solvents.

\subsection{Anisotropy g-factor and molar ellipticity spectra}

$C D$ spectra for various analytes treated with one molar equivalent of ninhydrin hydrate were collected according to the CD sensing protocol reported above and plotted as anisotropy gfactor vs wavelength as previously described by Zonta. ${ }^{1}$

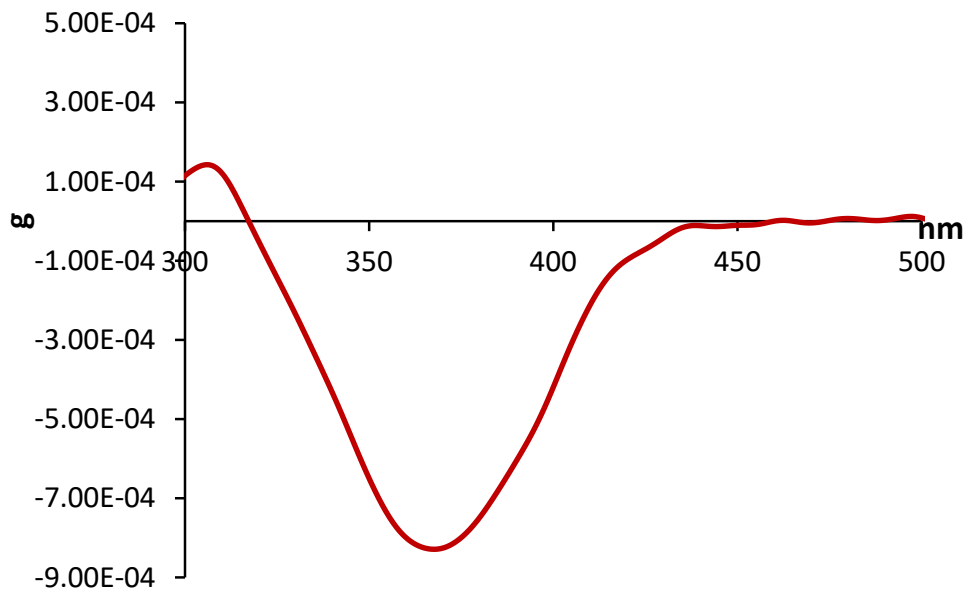

Figure S56. Sensing of with $(R)$-1-phenylethan-1-amine with $\mathbf{A}$ at $1.1 \mathrm{mM}$. 


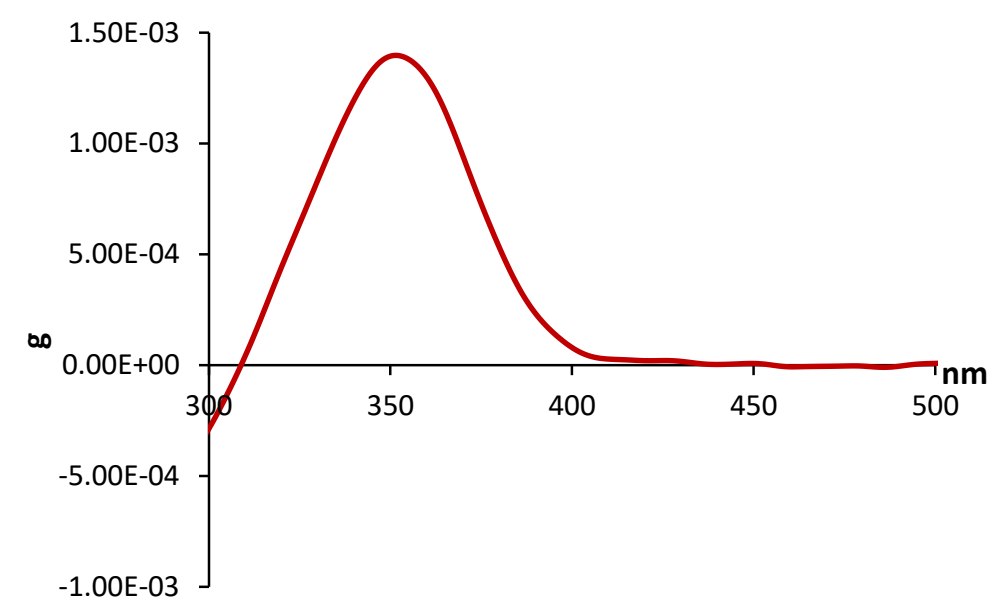

Figure S57. Sensing of $(1 R, 2 R)$-2-aminocyclohexan-1-ol with $\mathbf{A}$ at $1.1 \mathrm{mM}$. This analyte was obtained as $\mathrm{HCl}$ salt. The sensing experiments were performed in the presence of 1.0 equivalent of $\mathrm{Et}_{3} \mathrm{~N}$.

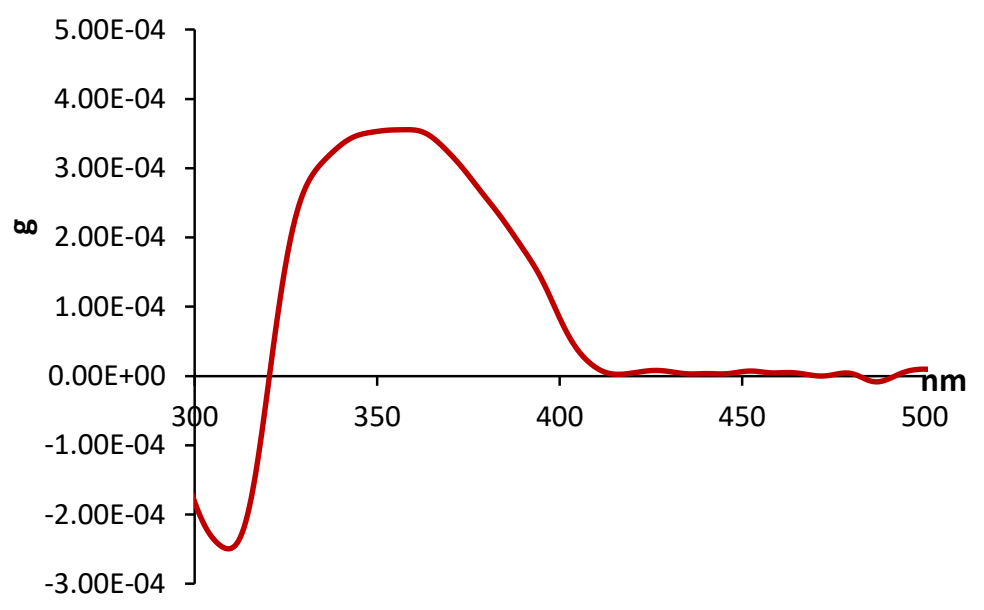

Figure S58. Sensing of $(1 R, 2 S)$-2-aminocyclohexan-1-ol with $\mathbf{A}$ at $1.1 \mathrm{mM}$. This analyte was obtained as $\mathrm{HCl}$ salt. The sensing experiments were performed in the presence of 1.0 equivalent of $\mathrm{Et}_{3} \mathrm{~N}$. 


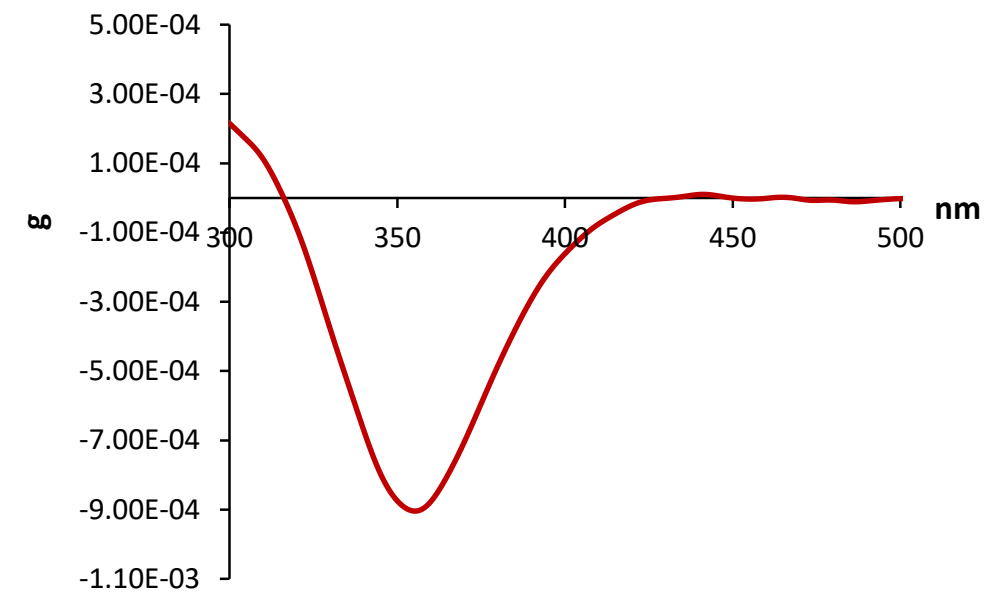

Figure S59. Sensing of $(R)$-3-aminopropane-1,2-diol with $\mathbf{A}$ at $1.1 \mathrm{mM}$.

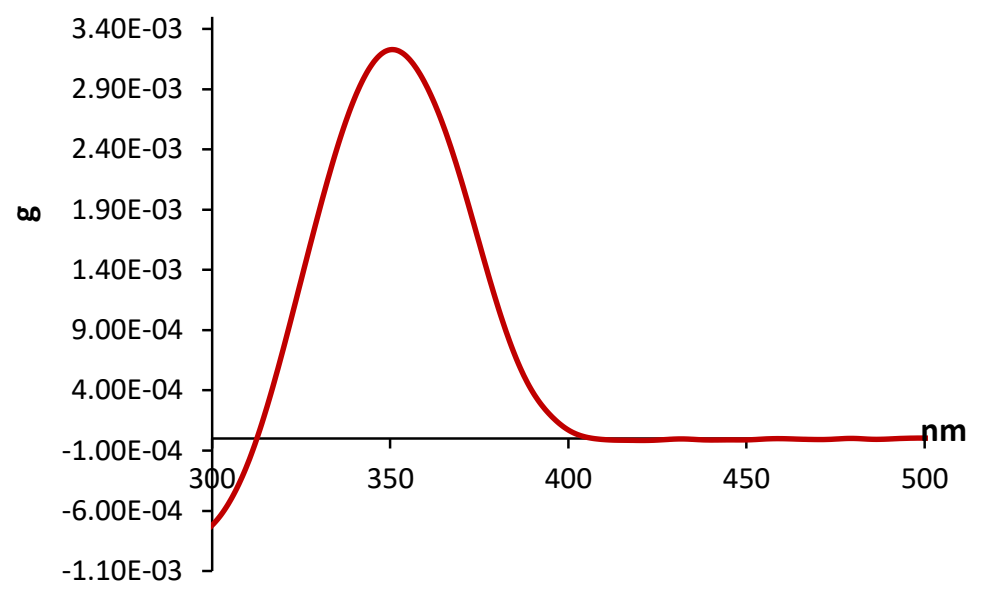

Figure S60. Sensing of $(R)$-pyrrolidin-2-ylmethanol with $\mathbf{A}$ at $1.1 \mathrm{mM}$.

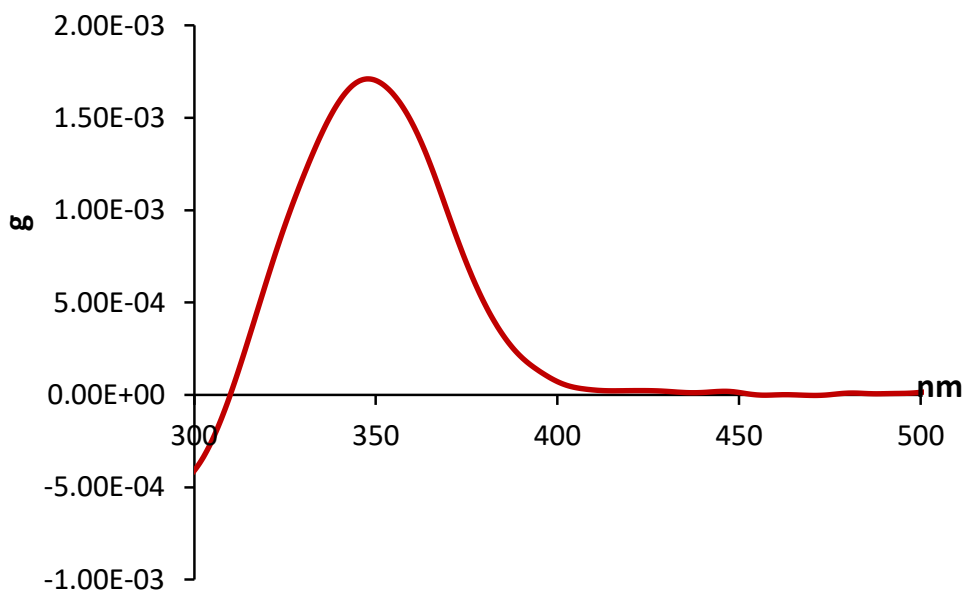

Figure S61. Sensing of $(R)$-2-amino-2-phenylethan-1-ol with A at $1.1 \mathrm{mM}$. 


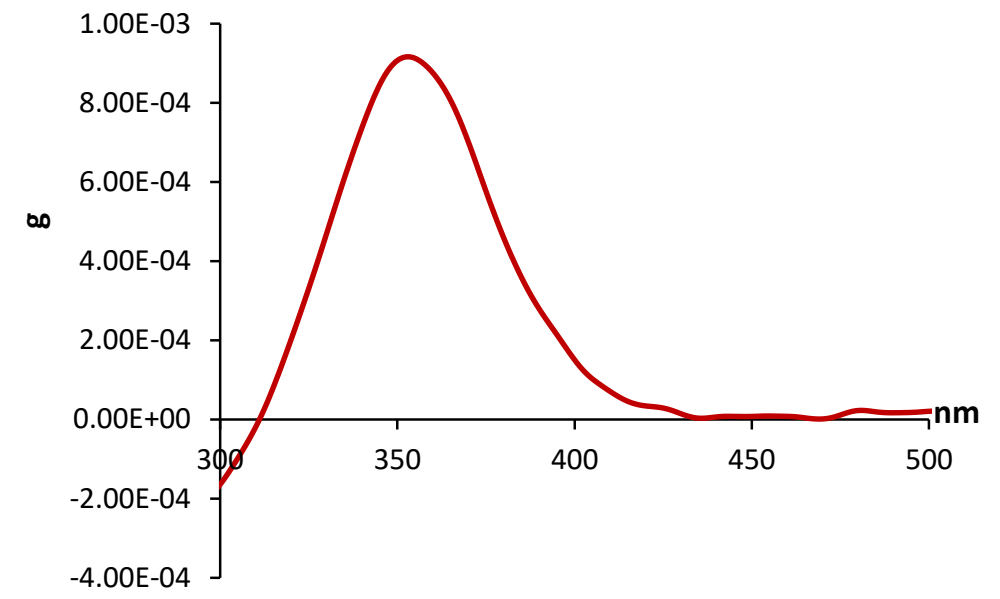

Figure S62. Sensing of $(R)$-2-amino-1-phenylethan-1-ol with $\mathbf{A}$ at $1.1 \mathrm{mM}$.

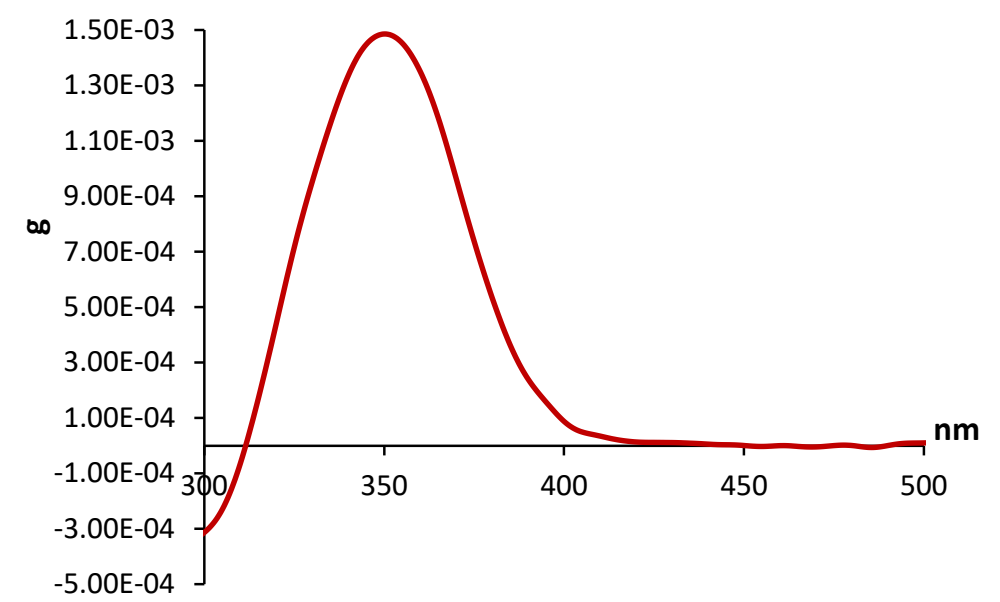

Figure S63. Sensing of (R)-2-amino-4-(methylthio)butan-1-ol with $\mathbf{A}$ at $1.1 \mathrm{mM}$.

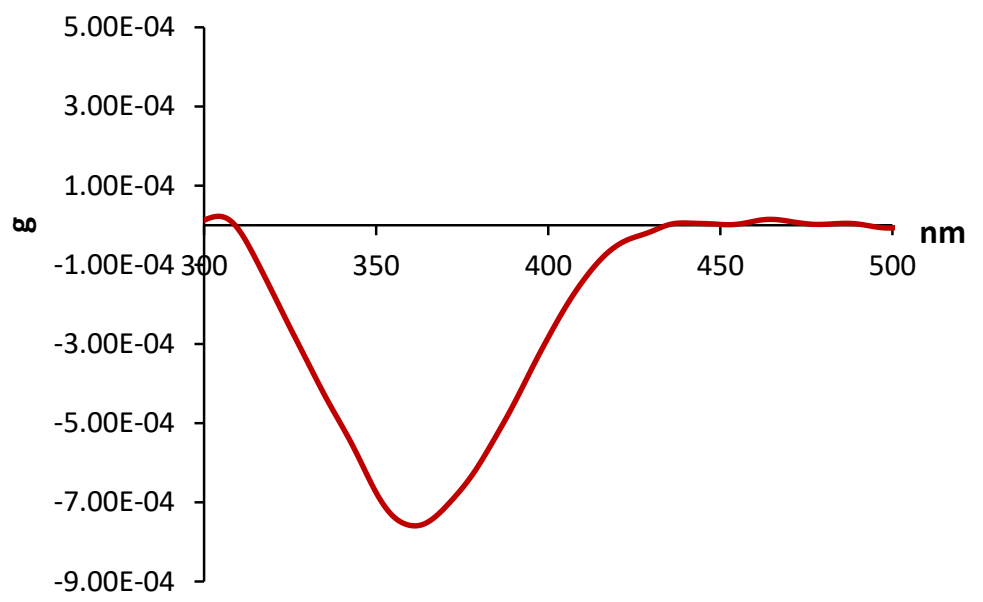

Figure S64. Sensing of $(R)$-1-cyclohexylethan-1-amine with $\mathbf{A}$ at $1.1 \mathrm{mM}$. 


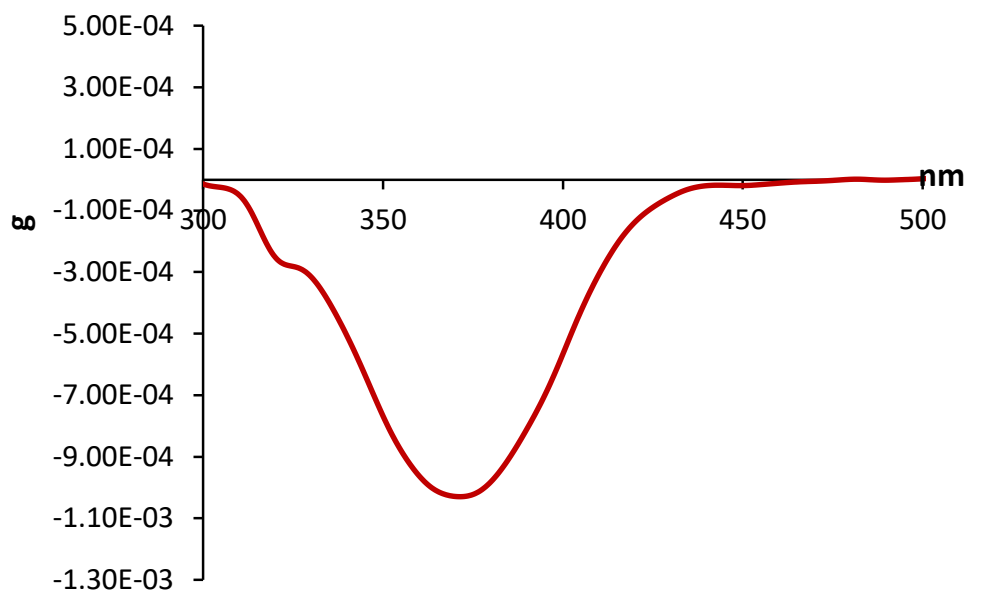

Figure S65. Sensing of $(R)$-1-(naphthalen-2-yl)ethan-1-amine with $\mathbf{A}$ at $1.1 \mathrm{mM}$.

Selected CD spectra of analytes treated with one molar equivalent of ninhydrin hydrate were collected according to the CD sensing protocol reported above and plotted as molar ellipticity, $\theta$, vs wavelength.

$\mathrm{MW}=$ molecular weight $(\mathrm{g} / \mathrm{mol})$

$$
\theta=\frac{\text { MW } \times \text { mdeg }}{10 \times \text { path length } \times c}
$$

mdeg=millidegrees $(C D)$

path length $=10 \mathrm{~cm}$

$\mathrm{c}=$ concentration in $(\mathrm{g} / \mathrm{L})$

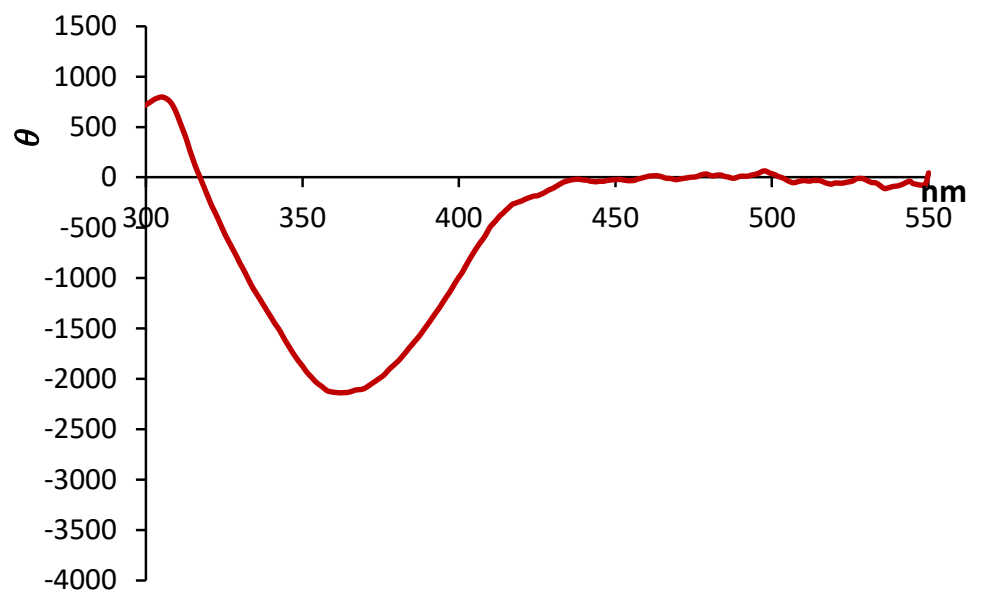

Figure S66. Sensing of $(R)$-1-phenylethan-1-amine with $\mathbf{A}$ at $1.1 \mathrm{mM}$. 


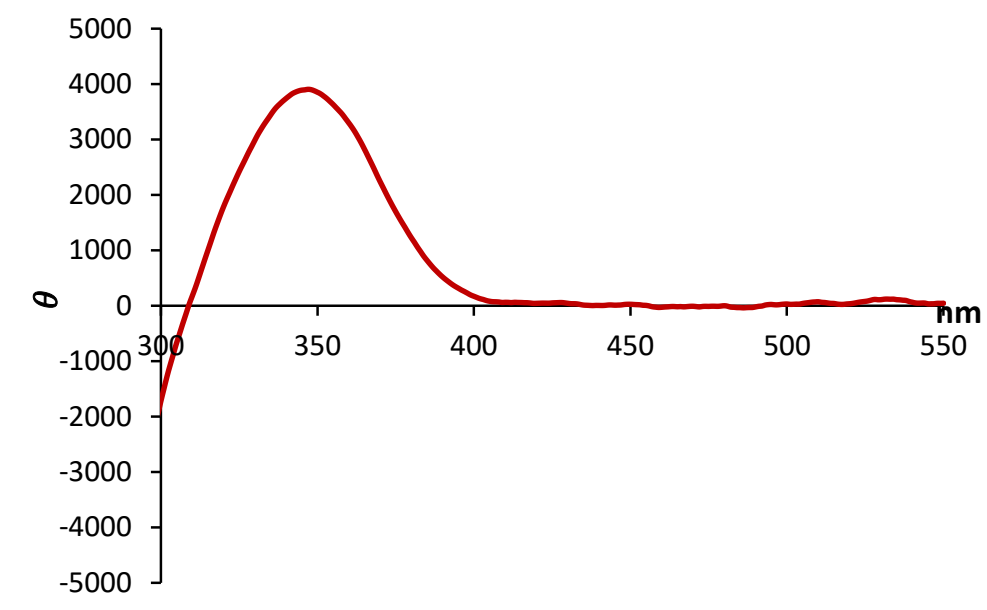

Figure S67. Sensing of $(1 R, 2 R)$-2-aminocyclohexan-1-ol with $\mathbf{A}$ at $1.1 \mathrm{mM}$. This analyte was obtained as $\mathrm{HCl}$ salt. The sensing experiments were performed in the presence of 1.0 equivalent of $\mathrm{Et}_{3} \mathrm{~N}$.

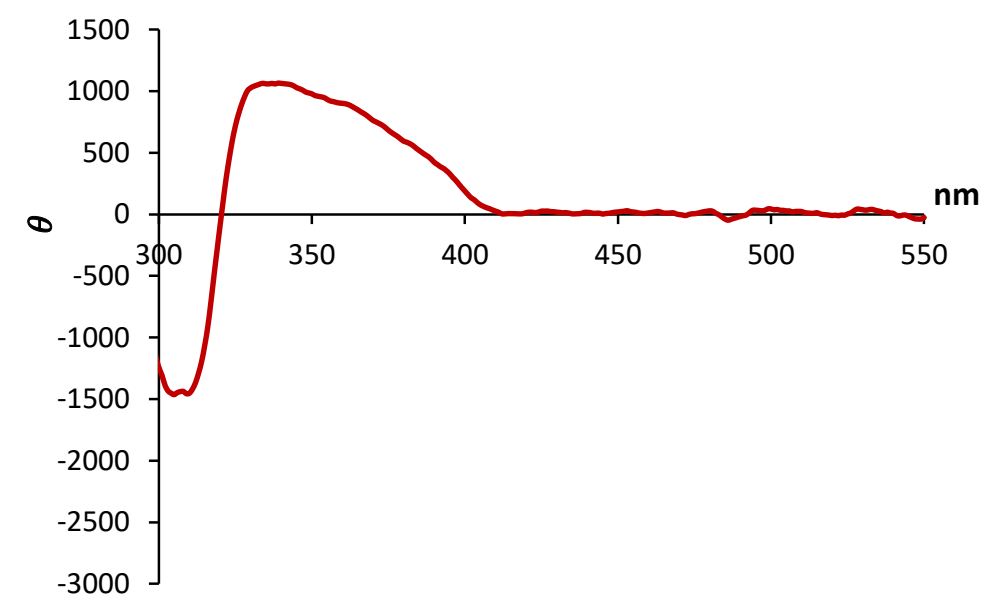

Figure S68. Sensing of $(1 R, 2 S)$-2-aminocyclohexan-1-ol with $\mathbf{A}$ at $1.1 \mathrm{mM}$. This analyte was obtained as $\mathrm{HCl}$ salt. The sensing experiments were performed in the presence of 1.0 equivalent of $\mathrm{Et}_{3} \mathrm{~N}$. 


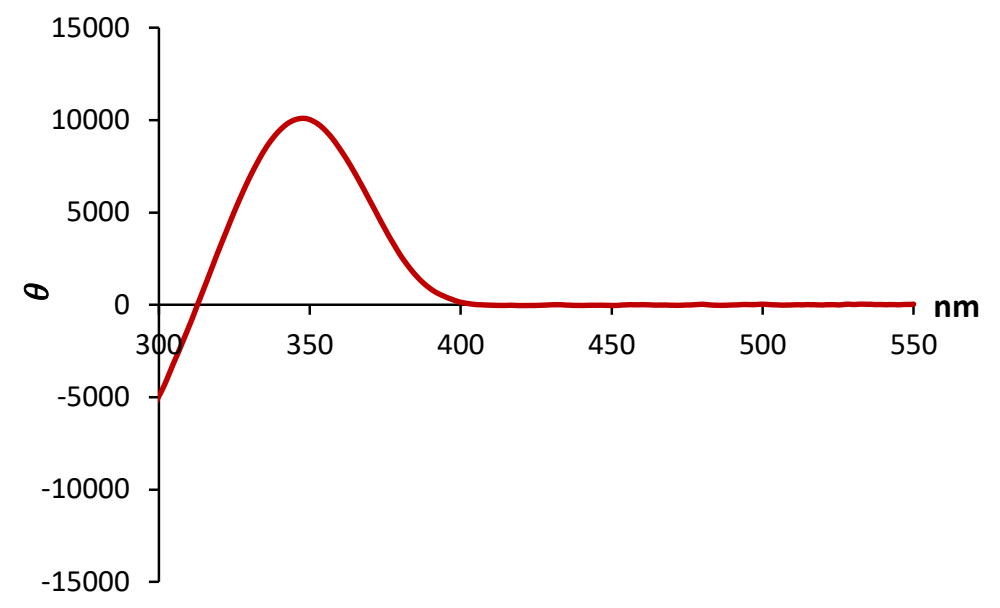

Figure S69. Sensing of $(R)$-pyrrolidin-2-ylmethanol with $\mathbf{A}$ at $1.1 \mathrm{mM}$.

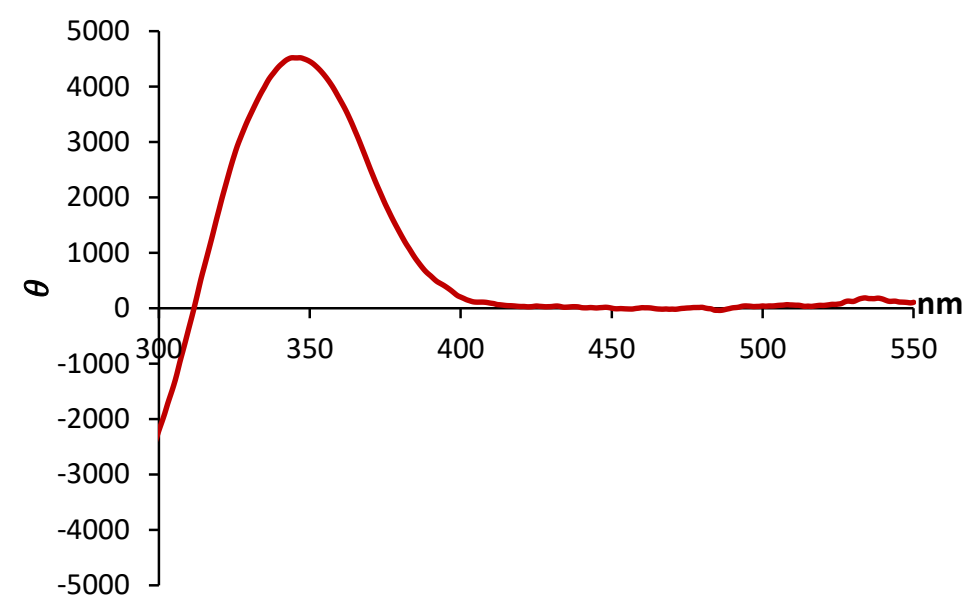

Figure S70. Sensing of (R)-2-amino-4-(methylthio)butan-1-ol with $\mathbf{A}$ at $1.1 \mathrm{mM}$.

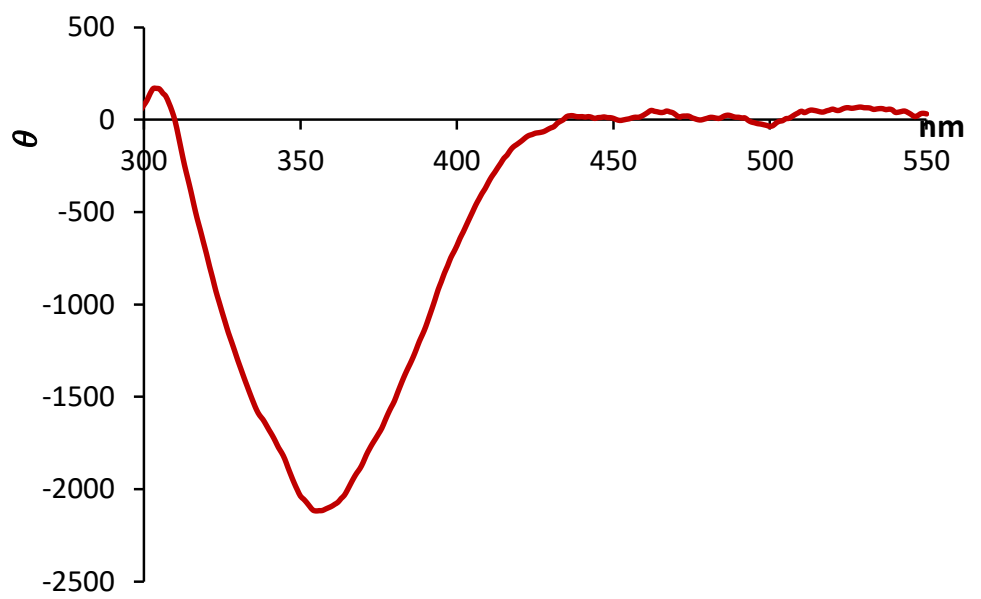

Figure S71. Sensing of $(R)$-1-cyclohexylethan-1-amine with $\mathbf{A}$ at $1.1 \mathrm{mM}$. 


\subsection{Dynamic covalent chemistry and equilibration study}

a) Study with phenylethan-1-amine 1 .

A solution of ninhydrin hydrate was prepared according to the above CD sensing protocol ( 5 $\mathrm{mM}$ ) with equimolar amounts of $(R)$-phenylethan-1-amine in DMSO. A reaction aliquot was then diluted to $1.1 \mathrm{mM}$ with $\mathrm{ACN}$ and subjected to $\mathrm{CD}$ sensing (red). Using a concentrated stock solution of $(S)$-phenylethan-1-amine, 0.2 equivalents of the $S$ enantiomer were added to the original $1.1 \mathrm{mM}$ mixture. The decline of the CD signal was monitored at 0.5 (blue), 10.0 (dashed orange), 20.0 (dotted green), and 30.0 (dark green) minutes. This equilibrated solution was then exposed to an additional 1.8 molar equivalents of $(S)$-phenylethan-1-amine and analyzed after 30 seconds(grey). The mixture then contained a total of 2.0 equivalents of the $S$ enantiomer and 1.0 equivalent of the $R$ enantiomer. CD measurements of the mixture $(1.1 \mathrm{mM}$ ninhydrin hydrate; $1.1 \mathrm{mM} R ; 2.2 \mathrm{mM} \mathrm{S}$ ) were taken at 10.0 minutes (yellow), 20.0 minutes (light blue), and 30.0 minutes (dotted red).
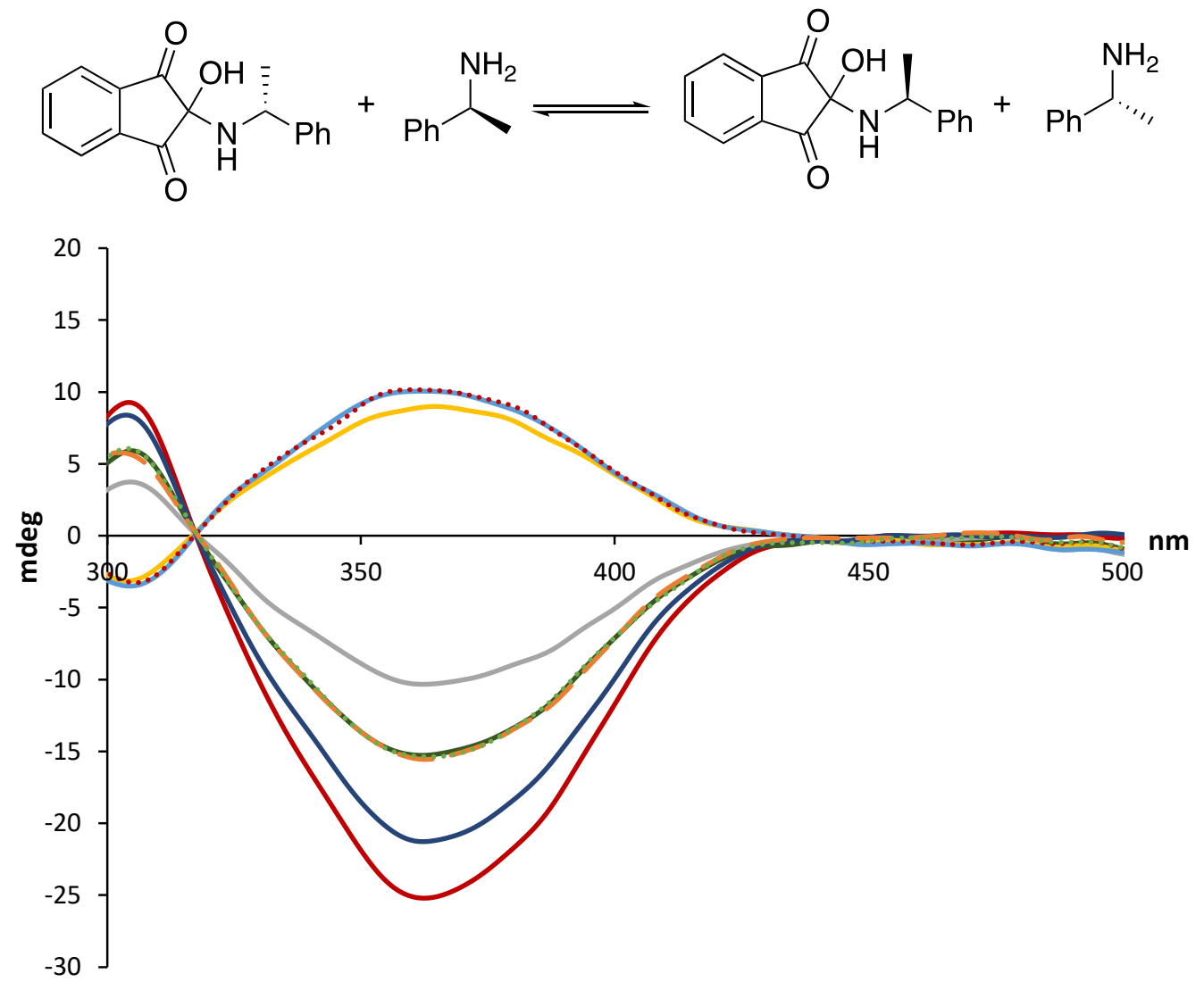

Figure S72. CD sensing of $(R)-\mathbf{1}$ with $\mathbf{A}$ and subsequent addition of $(S)-\mathbf{1}$. 
b) Study with 2-methylpyrollidine 10.

A solution of ninhydrin hydrate was prepared according to the above CD sensing protocol ( 5 $\mathrm{mM}$ ) and diluted to $1.1 \mathrm{mM}$ for CD measurement with equimolar ( $R$ )-2-methylpyrollidine (red). An equimolar amount of $(S)$-2-methylpyrollidine $(2.2 \mu \mathrm{L}, 125.0 \mathrm{mM})$ was added to the original $1.1 \mathrm{mM}$ sensing solution and a CD spectrum was obtained immediately (black). The dynamic covalent chemistry and rapid amine exchange results in the formation of a 1:1 equilibrium between $(R)-10 A$ and $(S)-10 A$ which is CD-silent.
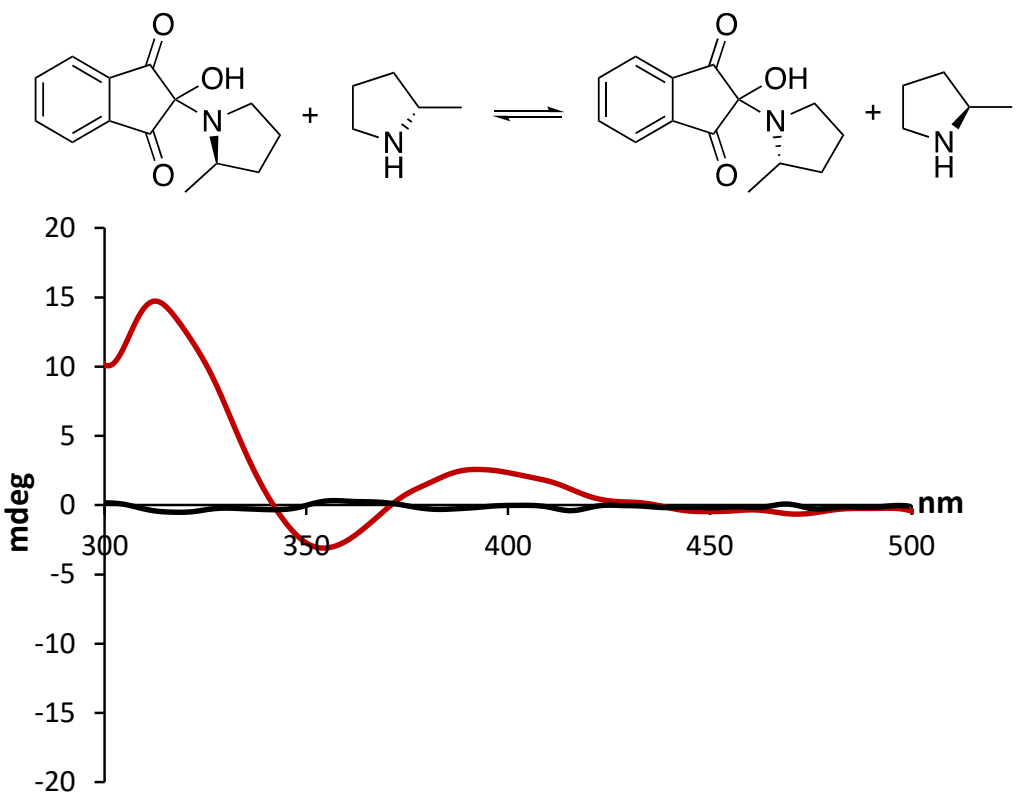

Figure S73. CD sensing of $(R)-10$ with $\mathbf{A}$ and subsequent addition of $(S)-10$. 


\section{Quantitative ee determination}

A solution of $\mathbf{A}(10.0 \mathrm{mM})$ was prepared in DMSO and $500.0 \mu \mathrm{L}$ amounts were distributed into vials containing $500.0 \mu \mathrm{L}$ of DMSO to generate reaction concentrations of $5.00 \mathrm{mM}$. Equimolar amounts of 1-phenylethan-1-amine dissolved in DMSO with varied ee values $(100.0,80.0,60.0$, $40.0,20.0,0,-20.0,-40.0,-60.0,-80.0,-100.0 \%$ ee) were subsequently added $(0.005 \mathrm{mmol}, 10$ $\mu \mathrm{L})$. CD measurements were then taken by dispensing $550 \mu \mathrm{L}$ aliquots of the reaction mixtures into a quartz cuvette and dilution to $2.5 \mathrm{~mL}$ with acetonitrile to afford an ee calibration curve. A second set of ninhydrin sensing reactions were prepared following the same procedure described using random ee mixtures of the same amine $(72.0,50.0,38.0,-16.0,-46.0,-84.0 \%$ ee).

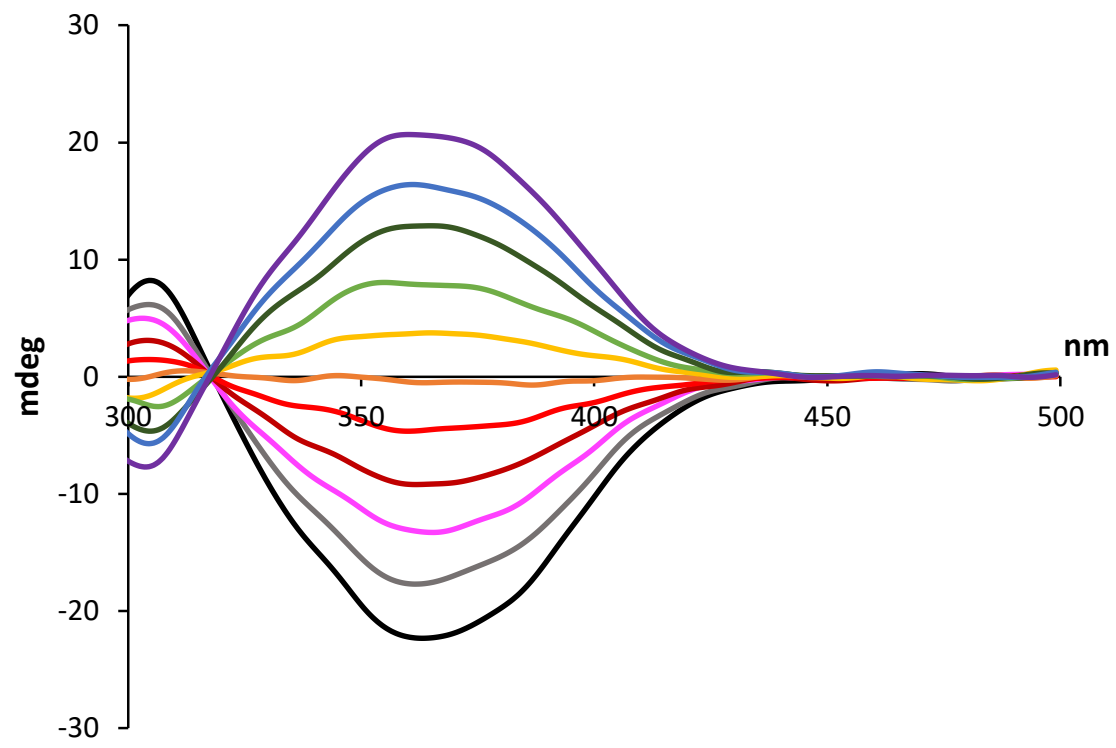

Figure S74. CD spectra obtained with varied enantiomeric compositions of 1-phenylethan-1amine and $\mathbf{A}$ at $1.1 \mathrm{mM}$.

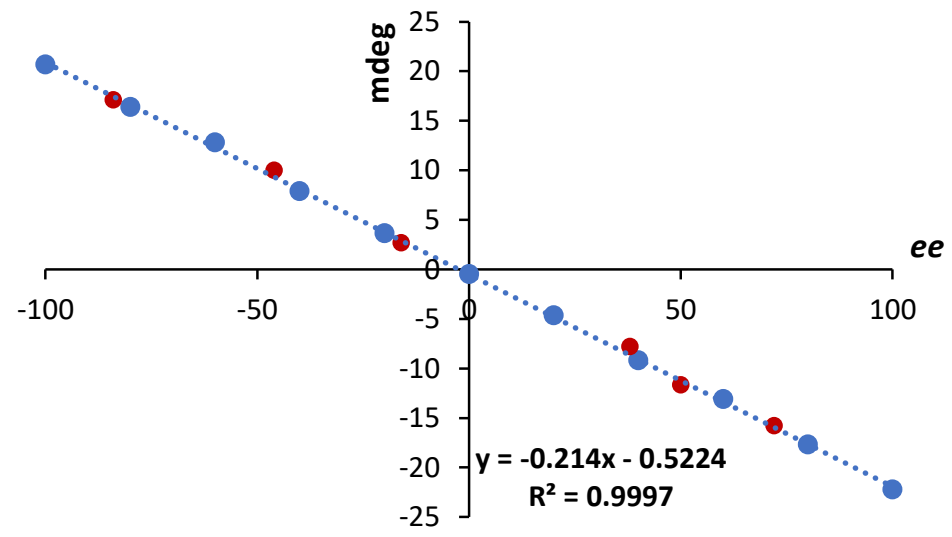

Figure S75. Linear correlation between the chiroptical responses of $\mathbf{A}$ at $360 \mathrm{~nm}$ and the analyte $e e^{\prime}$ s (blue) and sensing of random ee compositions of 1-phenylethan-1-amine samples (red). 
Table S1. Comparison of ee values of randomly prepared enantiomeric mixtures of 1phenylethan-1-amine with the sensing results. Positive ee values refer to samples enriched in $(S)-1$ and negative values to the samples with excess of the $(R)$-enantiomer.

\begin{tabular}{cc} 
Actual sample (\% ee) & Sensing results (\% ee) \\
\hline 72.0 & 71.2 \\
50.0 & 52.0 \\
38.0 & 34.0 \\
-16.0 & -15.0 \\
-46.0 & -49.2 \\
-84.0 & -82.4
\end{tabular}

\section{References}

1. Zardi, P.; Wurst, K.; Licini, G.; Zonta, C. Concentration-Independent Stereodynamic g-Probe for Chiroptical Enantiomeric Excess Determination. J. Am. Chem. Soc. 2017, 139, 15616-15619. 